\title{
GROUPS WITH THE SAME LOWER CENTRAL SEQUENCE AS A RELATIVELY FREE GROUP. II. PROPERTIES
}

\author{
BY \\ GILBERT BAUMSLAG $\left({ }^{1}\right)$
}

\section{0 . Introduction.}

0.0. This paper is a continuation of [3], which contains the motivation for this work. Many of the terms and notations used in [3] will be used throughout this paper without any explicit reference.

Suppose that $\mathfrak{B}$ is a variety of groups. A group $P$ is termed parafree $\left({ }^{2}\right)$ in $\mathfrak{B}$, or a parafree $\mathfrak{B}$-group, or simply parafree if there is no question as to which variety is involved, if

(i) $P$ is a $\mathfrak{B}$-group i.e., $P \in \mathfrak{B}$;

(ii) $P$ is residually nilpotent;

(iii) $P$ has the same lower central sequence $\left({ }^{2}\right)$ as some free $\mathfrak{B}$-group.

The object of this paper is to investigate the properties of such parafree groups with the basic hope (which is amply fulfilled) that many properties of the relevant free group persist in the corresponding parafree group.

0.1. The first section is preliminary in nature being essentially elementary. First we prove that if $P$ is a parafree $\mathfrak{B}$-group of finite rank and if $N$ is a normal subgroup of $P$ whose quotient $P / N$ is again parafree in $\mathfrak{B}$ of the same rank as $P$, then $N=1$. This almost obvious fact implies that parafree groups of finite rank are hopfian. Of course parafree groups of rank one are cyclic. It follows easily from this that a parafree group of rank two is freely decomposable if and only if it is free. Next we consider absolutely parafree groups i.e., groups parafree in the variety of all groups. For such groups we prove that free products and free factors are again parafree.

0.2 . In $\$ 2$ we complement the results in [3] by obtaining some more, rather different, parafree groups. We first consider one-relator groups.

THEOREM 2.1. Let

$$
H_{i, j}=\left(a, b, c ; a=\left[c^{i}, a\right]\left[c^{j}, b\right]\right) .
$$

Then $H_{i, j}$ is (absolutely) parafree but not free whenever $i j \neq 0$. Moreover if $F$ is an absolutely free group of rank two then

$$
H_{i, j} /\left[H_{i, j}, H_{i, j}^{\prime \prime}\right] \cong F /\left[F, F^{\prime \prime}\right]
$$

(where $X^{\prime \prime}$ is the second derived group of the group $X$ ).

Received by the editors September 3, 1968.

(1) Support from the National Science Foundation is gratefully acknowledged.

(2) See [3]. 
Theorem 2.1 was announced in [4]. Whether the parameters $i$ and $j$ distinguish the groups $H_{i, j}$ is unknown at present. However S. Meskin [27] has made some inroads into this question.

Next we consider, in $\$ 2$, ascending unions of parafree groups (in an arbitrary variety $\mathfrak{B}$ ) and establish conditions for such unions to have certain properties such as parafreeness. This leads for example to the following rather remarkable theorem (which was announced in [5]).

THEOREM 2.2. Let $\mathfrak{B}_{1}, \mathfrak{B}_{2}, \ldots$ be any countable family of varieties of groups, none of which is the variety of all groups. Then there exists an absolutely parafree group $P$ of rank two with the following properties:

(1) $P$ is an ascending union of free groups of rank two (and hence is locally free);

(2) $P / V_{i}(P) \cong F / V_{i}(F)$ for $i=1,2, \ldots$, where $F$ is absolutely free of rank two (and $V_{i}(X)$ is the smallest normal subgroup of the group $X$ such that $\left.X / V_{i}(X) \in \mathfrak{B}_{i}\right)$;

(3) $P$ is not free.

The difference between Theorem 2.1 and Theorem 2.2 is that the $H_{i, j}$ are finitely generated although $P$ is not. It should also be pointed out that $P$ has finite rank but infinite deviation.

0.3. In $\$ 3$ we turn our attention to some embedding theorems for parafree groups in certain well-behaved varieties, the so-called Magnus varieties (see [3]). It follows from A. I. Mal'cev [26] that a parafree group in a Magnus variety $\mathfrak{M}$ has a representation in a graded algebra $A$. If $\mathfrak{M}$ is the variety of all groups, $A$ is a power-series algebra. On the other hand if $\mathfrak{M}$ is the variety of all metabelian groups then $A$ can be chosen in a rather nice way. This yields in particular the pleasing

COROLlARY. Every parafree group in the variety of all metabelian groups has a faithful representation in a matrix ring over a commutative field.

Whether every absolutely parafree group admits such an embedding is an open question.

We prove last in $\$ 3$ a generalization of a theorem about absolutely free groups.

THEOREM 3.3. Every absolutely parafree group of countable rank can be embedded in a parafree group of rank two.

Theorem 3.3 indicates to some extent the complicated nature of parafree groups of rank two since there are at least continuously many parafree groups of countable rank (see [3]).

0.4 . In $\S 4$ we turn our attention to the subgroup structure of parafree groups. We begin by first proving the (easy)

THeORem 4.1. Let $P$ be a parafree group in a Magnus variety $\mathfrak{M}$ and let $X$ be $a$ subset of $P$ which freely generates a free abelian $\mathfrak{M}$-group modulo $\gamma_{2} P$. Then $X$ freely generates a free $\mathfrak{M}$-group. 
Actually Theorem 4.1 follows almost immediately from [6]. The other theorems in $\S 4$ are based on the embedding theorem of $\S 3$ and the connection between the algebra $A$ and an associated graded algebra (see $\$ 3$ ). The most pleasing subgroup theorem occurs in the absolute case.

THEOREM 4.2. The two-generator subgroups of an absolutely parafree group are free and so are the abelian subgroups.

It follows from Theorem 4.2 that the center of an absolutely parafree group is either the identity subgroup or the whole group.

Similar results hold also for parafree groups in the variety of all metabelian groups. For example we shall prove

THEOREM 4.3. Let $P$ be parafree in the variety of all metabelian groups. Then the two-generator subgroups of $P$ are isomorphic to two-generator subgroups of free metabelian groups.

0.5 . In $\$ 5$ we turn our attention to the residual properties of parafree groups again making use of the embedding theorems of $\$ 3$. Our main results here are

THEOREM 5.1. Parafree groups are residually parafree-of-finite-rank.

THEOREM 5.2. A noncyclic absolutely parafree group is residually parafree of rank two.

THEOREM 5.3. A noncyclic parafree group in the variety of all metabelian groups is residually parafree of rank two.

One might suspect from these theorems that absolutely parafree groups are residually-finitely-generated. This is not the case, however, as we shall show by an example.

We shall need another (somewhat easier to prove) residual property of parafree groups in connection with the study of Frattini subgroups of parafree groups (in §7), viz. :

THEOREM 5.4. Let $\mathfrak{B}$ be a variety of groups and suppose that $\mathfrak{B}$ is the union of its subvarieties $\mathfrak{B}_{\lambda}(\lambda \in \Lambda)$. Then a parafree group $P$ in $\mathfrak{B}$ is residually a parafree group in $\left\{\mathfrak{B}_{\lambda} \mid \lambda \in \Lambda\right\}$ i.e., if $a \in P(a \neq 1)$ there is a $\lambda \in \Lambda$, a parafree group $Q$ in $\mathfrak{B}_{\lambda}$ and $a$ homomorphism $\phi$ of $P$ onto $Q$ such that $a \phi \neq 1$.

0.6. In $\S 6$ we discuss some miscellaneous properties of parafree groups in product varieties of the form $\mathfrak{A} \mathfrak{B}$ where $\mathfrak{A}$ is the variety of all abelian groups and $\mathfrak{B}$ is any variety.

Our first aim is to obtain some information about elements of finite order in parafree groups. To this end let $F$ be the free group of countably infinite rank in a variety $\mathfrak{B}$. Define

$$
\gamma_{\omega} F=\bigcap_{n=1}^{\infty} \gamma_{n} F .
$$


Then we define $\mathfrak{B}^{\circ}$ to be the variety generated by $F / \gamma_{\omega} F$. Clearly $P$ is parafree in $\mathfrak{B}$ if and only if $P$ is parafree in $\mathfrak{B}^{\circ}$. Now it seems likely that if the free groups in a variety $\mathfrak{B}$, where $\mathfrak{B}=\mathfrak{B}^{\circ}$, are torsion-free then the parafree groups in $\mathfrak{B}$ are torsionfree. The varieties in which the free groups are torsion-free include the product varieties $\mathfrak{A} \mathfrak{U}$ (G. Higman [16]). We shall prove, in $\S 6$, the

THEOREM 6.1. Let $\mathfrak{U}$ be any variety of groups. Then the parafree groups in $\mathfrak{2} \mathfrak{U}$ are torsion-free.

For each integer $n>0$ let $\mathfrak{A}_{n}$ denote the variety of all abelian groups of exponent dividing $n$. Then the free groups in the product variety $\mathfrak{A O}_{n}$ are of a particularly simple kind. This is by no means the case for the corresponding parafree groups. Indeed we shall prove, also in $\S 6$, the following rather surprising theorems.

THEOREM 6.2. There are continuously many nonisomorphic groups in $\mathfrak{U A}_{2}$ of rank two.

THEOREM 6.3. There exists in $\mathfrak{A R}_{2}$ a parafree group of rank two whose cardinality is that of the continuum.

0.7. G. Higman and B. H. Neumann proved in [18] that absolutely free groups have trivial Frattini subgroups. More recently P. J. Cossey and A. Whittemore [13] have proved that in a great many product varieties of the form $\mathfrak{A B}$ (in particular when $\mathfrak{B}$ is a variety of locally finite $p$-groups) the free groups in $\mathfrak{2} \mathfrak{B}$ have trivial Frattini subgroup. We shall prove in $\S 7$ the

THEOREM 7.1. If $\mathfrak{B}$ is any variety then the finitely generated parafree groups in $\mathfrak{A B}$ have trivial Frattini subgroup.

An easy consequence of Theorem 7.1 is the

COROLlary 1. Finitely generated absolutely parafree groups have trivial Frattini subgroup.

Curiously enough the requirement in Theorem 7.1 that the parafree groups are finitely generated is actually necessary. For we shall construct, in $\$ 7$, a parafree group in $\mathfrak{X}_{2}$ with nontrivial Frattini subgroup. However I do not know whether the restriction to finitely generated absolutely parafree groups in Corollary 1 is also necessary.

\section{Elementary properties.}

1.1. We prove first

THEOREM 1.1. Let $P$ be parafree of finite rank and suppose $P / N$ is parafree of the same finite rank as $P$. Then $N=1$.

Proof. Since $P$ and $P / N$ have the same rank,

$$
P / \gamma_{n} P \cong P /\left(\gamma_{n} P\right) N \quad\left(\cong P / N / \gamma_{n}(P / N)\right)
$$


for every $n$. Now finitely generated nilpotent groups are hopfian. Hence $N \leqq \gamma_{n} P$ for every $n$. Therefore $N=1$.

COROLlaRY 1. Parafree groups of finite rank are hopfian.

Corollary 1 is the obvious analogue (indeed generalization) of the corresponding result for absolutely free groups. Whether every parafree group of infinite rank is, like the corresponding free group, nonhopfian is as yet unknown.

1.2. A residually nilpotent group $G$ with cyclic commutator factor group $G / \gamma_{2} G$ is cyclic since then $\gamma_{2} G=\gamma_{3} G=\cdots$. It follows from this remark and the comments in the introduction to [3] that

LEMMA 1.1. A parafree group that can be generated by two elements is free.

We use Lemma 1.1 to prove the following theorem which highlights a striking difference between free groups and parafree groups.

THEOREM 1.2. A parafree group $P$ of rank two in a variety $\mathfrak{B}=\mathfrak{B}^{\circ}$ is freely decomposable (in the obvious varietal sense) if and only if either $P$ is free or $\mathfrak{B}$ is of finite but not prime-power exponent.

Proof. Let $A$ and $B$ be nontrivial groups in $\mathfrak{B}$ and suppose $P$ is the free product (in $\mathfrak{B}$ ) of $A$ and $B$. Then

$$
P / \gamma_{2} P \cong A / \gamma_{2} A \times B / \gamma_{2} B
$$

If $P$ is either of exponent zero or of prime-power exponent then both $A / \gamma_{2} A$ and $B / \gamma_{2} B$ are cyclic. But then $A$ and $B$ are themselves cyclic. So $P$ is free.

If $P$ is of finite exponent then $P$ is a direct product of its sylow $p$-subgroups and hence the free product (in $\mathfrak{B}$ ) of these sylow p-subgroups (cf., e.g., [28]).

1.3. We specialize now, in this paragraph, to the variety of all groups.

THEOREM 1.3. Free products and free factors of absolutely parafree groups are parafree. Furthermore if $A$ is absolutely parafree of rank $r$ and $B$ is absolutely parafree of rank $s$ then their free product is absolutely parafree of rank $r+s$.

Proof. The free product of residually torsion-free nilpotent groups is residually (torsion-free) nilpotent [26]. So the free product of parafree groups is residually nilpotent. But if the groups $\bar{A}$ and $\bar{B}$ are free nilpotent of class $c$ then, modulo the $(c+1)$ th term of its lower central series, the free product of $\bar{A}$ and $\bar{B}$ is also free nilpotent of class $c$. It follows easily that the free product of parafree groups is parafree.

Conversely suppose $P$ is parafree and that $P$ is the free product of $A$ and $B$. Then

$$
P / \gamma_{2} P \cong A / \gamma_{2} A \times B / \gamma_{2} B
$$

So we can find subsets $X$ of $A$ and $Y$ of $B$ respectively such that $X \cup Y$ freely generates $P$ modulo $\gamma_{2} P, X$ freely generates $A$ modulo $\gamma_{2} A$ and $Y$ freely generates $B$ modulo $\gamma_{2} B$. 
Now $P / \gamma_{n} P$ is a free nilpotent group freely generated by $X \cup Y$ modulo $\gamma_{n} P$. Therefore it follows from [6] that $X$ freely generates a free nilpotent group modulo $\gamma_{n} P$. Hence $X$ freely generates a free nilpotent group modulo $\gamma_{n} A$. So $A$ has the same lower central sequence as a free group. But $A$ is residually nilpotent since $P$ is. So $A$ is parafree.

The last remark about the rank of a free product now follows immediately. This completes the proof of Theorem 1.3.

2. Some surprising examples of parafree groups.

2.1. Our first objective in this section is to prove

THEOREM 2.1. Let $i$ and $j$ be nonzero integers, let

$$
H=\left(a, b, c ; a=\left[c^{i}, a\right]\left[c^{j}, b\right]\right)
$$

and let $F$ be a free group of rank two. Then $H$ is parafree. Moreover

(i) $H$ (like $F$ ) is the third term of an exact sequence $1 \rightarrow N \rightarrow H \rightarrow Z \rightarrow 1$ where $N$ is free and $Z$ is infinite cyclic;

(ii) $H /\left[H^{\prime \prime}, H\right] \cong F /\left[F^{\prime \prime}, F\right]$;

(iii) $H$ is not free.

2.2. In order to prove Theorem 2.1 we employ the Reidemeister-Schreier procedure for obtaining generators and defining relations for a subgroup of a group given by generators and defining relations (see e.g., Magnus, Karrass and Solitar [25]). Thus, denoting the normal subgroup (of a group $Y$ ) generated by a subset $X$ by $\operatorname{gp}_{Y}(X)$, we put

$$
N=\operatorname{gp}_{H}(a, b)
$$

By Reidemeister-Schreier

$$
N=\left(\ldots, a_{-1}, a_{0}, a_{1}, \ldots, \ldots, b_{-1}, b_{0}, b_{1}, \ldots ; \ldots, a_{n}=a_{n+i}^{-1} a_{n} b_{n+j}^{-1} b_{n}, \ldots\right)
$$

(where the subscript $n$ ranges over all integers). We may assume that $j>0$. It follows then that $N$ is a free group freely generated by the elements

$$
\ldots, a_{-1}, a_{0}, a_{1}, \ldots, b_{0}, b_{1}, \ldots, b_{j-1} \text {. }
$$

But obviously $H / N$ is free cyclic. So we have established (i) of Theorem 2.1.

2.3. In order to verify (ii) we proceed as follows. First notice that

$$
a=\left[c^{i}, a\right]\left[c^{j}, b\right]=c^{-i} a^{-1} c^{i} a\left[c^{j}, b\right]=a c^{-i} a^{-1} c^{i} \cdot\left[c^{-i} a^{-1} c^{i}, a\right]\left[c^{j}, b\right] .
$$

Hence $c^{-i} a c^{i}=\left[c^{-i} a^{-1} c^{i}, a\right]\left[c^{j}, b\right]$. This in turn implies, using the customary exponential notation for conjugation, that

$$
a=\left[a^{-c^{1}}, a\right]^{c^{-1}}\left[c^{j}, b\right]^{c^{-1}} \text {. }
$$

Now $a$ clearly lies in $H^{\prime}$. So $\left[a^{-c^{1}}, a\right]^{c^{-1}} \in H^{\prime \prime}$ and hence $\left[a^{-c^{1}}, a\right]^{c^{-1}}$ is central modulo $\left[H^{\prime \prime}, H\right]$. Therefore, modulo $\left[H^{\prime \prime}, H\right]$, we have

$$
a=\left[c^{i}, a\right]\left[c^{j}, b\right]=\left[c^{i},\left[a^{-c^{i}}, a\right]^{-c^{i}}\left[c^{j}, b\right]^{c^{-i}}\right]\left[c^{j}, b\right]=\left[c^{i},\left[c^{j}, b\right]^{c^{-1}}\right]\left[c^{j}, b\right] .
$$


If we put $\bar{H}=H /\left[H, H^{\prime \prime}\right], \bar{a}=a\left[H, H^{\prime \prime}\right], \bar{b}=b\left[H, H^{\prime \prime}\right]$ and $\bar{c}=c\left[H, H^{\prime \prime}\right]$, then $\bar{H}$ may be thought of as a 1-relator group in the variety $\mathfrak{B}$ of all groups whose center contains the derived group. Now the two-generator free groups in $\mathfrak{B}$ are residually nilpotent (J. Ridley [31]). So it follows from the proof of the corresponding theorem for absolutely free groups (see [25, Corollary 5.14.2, p. 354]), that $\bar{H}$ is free in $\mathfrak{B}$ of rank two. Thus we have proved (ii).

2.4. The proof of (iii) is a straightforward application of the algorithm of J. H. C. Whitehead [33] whereby one can effectively determine whether a group with a given single defining relation is free.

2.5. We are left with the proof that $H$ is parafree. Now if $\tilde{F}$ is the absolutely free group on $a, b, c$, then we can think of $H / \gamma_{n} H$ as the result of adding to $\tilde{F} / \gamma_{n} \tilde{F}$ the given relation

$$
a=\left[c^{i}, a\right]\left[c^{j}, b\right] .
$$

But $\tilde{F} / \gamma_{n} \tilde{F}$ is a free nilpotent group freely generated by $a \gamma_{n} \tilde{F}, b \gamma_{n} \tilde{F}$ and $c \gamma_{n} \tilde{F}$. It follows that $\tilde{F}$ is freely generated by

$$
a^{-1}\left[c^{i}, a\right]\left[c^{j}, b\right], b, c
$$

modulo $\gamma_{n} \widetilde{F}$ (since the Frattini subgroup of a nilpotent group contains the derived group). Consequently $H / \gamma_{n} H$ is certainly a free nilpotent group of rank two.

So the proof of the parafreeness of $H$ reduces to the proof that $H$ is residually nilpotent.

We begin by recalling that $H$ is a splitting extension of the free group $N$ by the infinite cyclic group gp. (c). It follows from the defining relations of $N$ that the elements $b_{n}(n=0,1,-1,2, \ldots)$ generate $N$ modulo $\gamma_{2} N$. So they generate $N$ modulo $\gamma_{c} N$ for every $c \geqq 1$. In addition the $b_{n}$ are independent modulo $\gamma_{2} N$. So they freely generate the free nilpotent group $N$ modulo $\gamma_{c} N$ for every $c$. Notice that $b_{n}^{c}=b_{n+1}$ for every integer $n$. The residual nilpotence of $H$ follows easily. For if $x \in H$ and $x \notin N$ then as $H / N$ is cyclic there is nothing to prove. So we may restrict attention to those elements $x \in H, x \neq 1, x \in N$. Since $N$ is free $x \notin \gamma_{l} N$ for some $l$. Without loss of generality we may assume that

$$
x \gamma_{l} N \in \operatorname{gp}\left(b_{0} \gamma_{l} N, \ldots, b_{2^{r}-1} \gamma_{l} N\right)
$$

for a suitably large choice of the integer $r$. Now let $M=\mathrm{gp}_{H}\left(c^{2^{r}}, \gamma_{l} N\right)$. Then $M N / M$ is a free nilpotent group freely generated by $b_{0} M, \ldots, b_{2^{r}-1} M$ and $H / M N$ is generated by $c M N$ and is of order $2^{r}$. Clearly $x \notin M N$. But a finitely generated torsion-free nilpotent group is residually a finite 2-group (K. W. Gruenberg [15]). So $H / M$ is itself residually a finite 2-group. Hence there is a normal subgroup $L$ of $H$ such that $x \notin L$ and $H / L$ is nilpotent. Therefore $H$ is residually nilpotent. This completes the proof of Theorem 2.1.

2.6. We shall establish now conditions under which ascending unions of parafree groups are again parafree. It is useful to define here the notion of parabasis 
for a parafree group $P$. Thus we term $B$, a subset of $P$, a parabasis for $P$ if $B$ freely generates $P$ modulo $\gamma_{2} P$. It follows that $B$ freely generates $P$ modulo $\gamma_{n} P$ for $n \geqq 2$.

We now prove

Proposition 2.1. Let $\mathfrak{B}$ be a Magnus variety and let

$$
A_{1} \subseteq A_{2} \subseteq \cdots \subseteq A_{n} \subseteq \cdots \subseteq A=\bigcup_{n=1}^{\infty} A_{n}
$$

be an ascending series of parafree groups (in $\mathfrak{B})$ of the same finite rank $k$. If $n_{\mathfrak{i}}=$ $\left|A_{i+1}\right| A_{i} \gamma_{2} A_{i+1} \mid<\infty$ for every $i=1,2, \ldots$, then

(i) $A$ is residually torsion-free nilpotent;

(ii) $A$ is residually a finite $p$-group if $p$ divides $n_{i}$ for only finitely many $i$;

(iii) $A$ is parafree if and only if $n_{i}=1$ for all but finitely many $i$.

Proof. Let $B_{2}$ be a parabasis for $A_{2}$, say $B_{2}=\{a, b, \ldots, c\}$. Then for a suitable choice of $m>0$,

$$
\left\{a^{m}, b^{m}, \ldots, c^{m}\right\} \subseteq A_{1} \quad \text { modulo } \gamma_{2} A_{2} \text {. }
$$

So if $B_{1}$ is a parabasis for $A_{1}, B_{1}$ must be independent modulo $\gamma_{2} A_{2}$. Therefore, by [6], $B_{1}$ freely generates modulo $\gamma_{l} A_{2}$ a free nilpotent $\mathfrak{B}$-group for every $l=2,3, \ldots$ By hopficity it follows that

$$
A_{1} \cap \gamma_{l} A_{2}=\gamma_{l} A_{1} \quad(l=2,3, \ldots) .
$$

Similarly we find that for $i \leqq j$

$$
A_{i} \cap \gamma_{l} A_{j}=\gamma_{l} A_{i} \quad(l=2,3, \ldots) .
$$

This immediately implies that

$$
A_{i} \cap \gamma_{l} A=\gamma_{l} A_{i} \quad(l=2,3, \ldots) .
$$

Now suppose $a \in A(a \neq 1)$. Then $a \in A_{i}$ for some $i$. Therefore $a \notin \gamma_{l} A_{i}$ for some $l$ since $A_{i}$ is residually nilpotent. By virtue of (1) this implies $a \notin \gamma_{l} A$.

But by virtue of (1), $A / \gamma_{l} A$ is a colimit of the torsion-free nilpotent groups $A_{i} / \gamma_{l} A_{i}, i=1,2, \ldots$. Therefore $A / \gamma_{l} A$ is torsion-free and we have proved (i).

In order to prove (ii) suppose $p$ is any prime which does not divide $n_{i}$ for $i=1,2, \ldots$ Define, for any group $G$,

$$
G(i, j)=\operatorname{gp}\left(x^{p^{i}} \mid x \in G\right) \gamma_{j} G .
$$

Then for each integer $r \leqq s$ and all $i, j$

$$
A_{\mathrm{r}} A_{s}(i, j)=A_{s} \text {. }
$$

Moreover it follows in much the same way as in the proof of (1) that

$$
A_{s}(i, j) \cap A_{r}=A_{r}(i, j),
$$

and hence that

$$
A(i, j) \cap A_{r}=A_{r}(i, j) .
$$


It follows immediately from (2) and the fact that $A_{r}$ is residually a finite $p$-group that $A$ too is residually a finite $p$-group.

Finally we prove (iii). Suppose first that $n_{i}>1$ for infinitely many $i$. Then by (1) $A / \gamma_{2} A$ is a colimit of the groups $A_{i} / \gamma_{2} A_{i}(i=1,2, \ldots)$. Each $A_{i} / \gamma_{2} A_{i}$ is a free abelian group of rank $k$. So $A / \gamma_{2} A$ is an ascending union of $k$-generator groups. Since this union is properly ascending $A / \gamma_{2} A$ is certainly not free abelian (since a free abelian group always has a maximal $k$-generator subgroup). So $A$ is not parafree. Conversely if $n_{i}=1$ for all but finitely many $i, A_{r}$ generates $A$ modulo $\gamma_{2} A$ for some $r$. Therefore, invoking (1), we have

$$
A / \gamma_{n} A=A_{r} \gamma_{n} A / \gamma_{n} A \cong A_{r} / \gamma_{n} A \cap A_{r}=A_{r} / \gamma_{n} A_{r}
$$

for every $n$. So by (i), $A$ is parafree. This completes the proof of Proposition 2.1.

It seems worthwhile to formulate one part of this proposition as

THEOREM 2.2. Let $A$ be the union of an ascending series $A_{1} \subseteq A_{2} \subseteq \cdots$ of parafree groups in a Magnus variety. If the $A_{i}$ are all of the same finite rank $k$ and the quotient groups $A_{i+1} / A_{i} \gamma_{2} A_{i+1}$ are finite for every $i=1,2, \ldots$, then $A$ is parafree if and only if $A_{i+1}=A_{i} \gamma_{2} A_{i+1}$ for all but finitely many $i$. Moreover in the event that $A$ be parafree its rank is $k$.

2.7. We shall now employ Theorem 2.2 to construct some really extraordinary parafree groups. We concentrate first on absolutely parafree groups.

THEOREM 2.3. Let $\mathfrak{B}_{1}, \mathfrak{B}_{2}, \ldots$ be any countable family of varieties of groups which does not include the variety of all groups. Then there exists an absolutely parafree group $P$ of rank two with the following properties:

(i) $P / V_{i}(P) \cong F / V_{i}(F)$ for $i=1,2, \ldots$, where $F$ is an absolutely free group of rank two;

(ii) $P$ is locally free;

(iii) $P$ is not free.

Proof. Let $\mathfrak{S}_{i}$ be the variety of all soluble groups of derived length at most $i$ and let

$$
\mathfrak{W}_{i}=\mathfrak{B}_{1} \cup \mathfrak{B}_{2} \cup \cdots \cup \mathfrak{B}_{i} \cup \mathfrak{S}_{i+1}
$$

for $i=1,2, \ldots$. Furthermore let $F_{i}=\operatorname{gp}\left(a_{i}, b_{i}\right)$ be the free group on $a_{i}$ and $b_{i}$.

Choose $u_{i} \in W_{i}\left(F_{i+1}\right)$ so that

$$
H_{i}=\operatorname{gp}\left(a_{i+1} u_{i}, b_{i+1}\right)
$$

is a proper subgroup of $F_{i+1}$ for $i=1,2, \ldots$ Note that if $F$ is a free group of rank two on $a$ and $b$ and if $u \in \gamma_{2} F$, then $a u$ and $b$ generate $F$ if and only if $u=\left[a, b^{i}\right]$ for some $i$ (this follows immediately from a theorem of J. Nielsen [30]). This makes the choice of $u_{i}$ particularly easy. Note also that since $u_{i} \in \gamma_{2} F_{i+1}, H_{i}$ is automatically a free subgroup of rank two. 
Let $\phi_{i}$ be the monomorphism from $F_{i}$ into $F_{i+1}$ defined by

$$
\phi_{i}: a_{i} \mapsto a_{i+1} u_{i}, b_{i} \mapsto b_{i+1} .
$$

Then $F_{i} \phi_{i}=H_{i}$ is a proper subgroup of $F_{i+1}$. Moreover the system of groups $F_{i}$ and homomorphisms $\phi_{i}$ provide us with a direct system (see e.g., Eilenberg and Steenrod [14]). Let $P$ be the colimit (direct limit) of this system:

$$
P=\operatorname{colt} F_{n} \text {. }
$$

$P$ may be viewed as the properly ascending union of its free subgroups $F_{n}$ :

$$
P=\bigcup_{n=1}^{\infty} F_{n}
$$

So $P$ is not finitely generated. Of course we may also write

$$
P=\bigcup_{n=1}^{\infty} F_{n}
$$

for every $i \geqq 1$.

We now compute $V_{i}(P)$ for $i \geqq 1,2, \ldots$. Clearly

$$
V_{i}(P)=\bigcup_{n=i}^{\infty} V_{i}\left(F_{n}\right)
$$

We shall prove that $V_{i}(P) \cap F_{n}=V_{i}\left(F_{n}\right)$ for $n \geqq i$. To do so let $F$ be the free group on $a$ and $b$ and consider the homomorphism $\theta_{i}$ from $F_{i}$ onto $F / V_{i}(F)$ defined by

$$
\theta_{i}: a_{i} \mapsto a V_{i}(F), b_{i} \mapsto b V_{i}(F) .
$$

Clearly the kernel of $\theta_{i}$ is $V_{i}\left(F_{i}\right)$. Now define $\theta_{i+1}: F_{i+1} \rightarrow F / V_{i}(F)$ by

$$
\theta_{i+1}: a_{i+1} \mapsto a V_{i}(F), b_{i+1} \mapsto b V_{i}(F) .
$$

Again the kernel of $\theta_{i+1}$ is $V_{i}\left(F_{i+1}\right)$. Notice that as $u_{i+1} \in W_{i+1}\left(F_{i+1}\right) \leqq V_{i}\left(F_{i+1}\right)$,

$$
\left(a_{i+1} u_{i+1}\right) \theta_{i+1}=a V_{i}(F)=a_{i} \theta_{i}
$$

and also that

$$
b_{i+1} \theta_{i+1}=b V_{i}(F)=b_{i} \theta_{i} .
$$

So $\theta_{i+1}$ agrees with $\theta_{i}$ on $F_{i}$. In this way we obtain a sequence of homomorphisms $\theta_{i}, \theta_{i+1}, \ldots$ each agreeing with its predecessor on the appropriate $F_{j}$. So the homomorphisms $\theta_{i}$ define in the obvious way a homomorphism $\theta$ of $P$ onto $F / V_{i}(F)$. Clearly the kernel of $\theta$ is the union of the kernels of the $\theta_{j}(j=i, i+1, \ldots)$. So

$$
K=\bigcup_{n=i}^{\infty} V_{i}\left(F_{n}\right)=\bigcup_{n=1}^{\infty} V_{i}\left(F_{n}\right)=V_{i}(P)
$$

This gives us therefore the desired isomorphism $P / V_{i}(P) \cong F / V_{i}(F)$.

Finally we observe that $P$ is parafree of rank two by Theorem 2.2. Hence $P$ is not free since it is not finitely generated but $P / \gamma_{2} P$ is. 
At the present time only a countable infinity of distinct varieties are known to exist. Let $\mathfrak{B}_{1}, \mathfrak{B}_{2}, \ldots$ be the family of all known varieties excluding the variety of all groups. Then the $\mathfrak{B}_{\mathfrak{i}}$ include (among others) the varieties generated by every finite group. So the following corollary is an immediate consequence of Theorem 2.3 .

COROLlaRY 1. There exists an absolutely parafree group $P$ of rank two with the following properties:

(i) $P$ is the union of free groups of rank two (and hence is locally free);

(ii) $P$ has precisely the same finite quotient groups as an absolutely free group $F$ of rank two;

(iii) $P / V(P) \cong F / V(F)$ for every polynilpotent variety $\mathfrak{B}$ (see $K$. W. Gruenberg [15] for the relevant definition);

(iv) $P$ is not free;

(v) every finitely generated quotient group of $P$ can be generated by two elements.

Only (v) needs to be verified. Thus we note that

$$
P=\bigcup_{i=1}^{\infty} F_{i}
$$

If $N$ is any normal subgroup of $P$,

$$
P / N=\bigcup_{i=1}^{\infty} F_{i} N / N
$$

Since $P / N$ is finitely generated $P / N=F_{i} N / N$ for some $i$. Therefore $P / N$ is a twogenerator group.

It is perhaps worthwhile emphasizing that we have constructed absolutely parafree groups of finite rank and infinite deviation. This answers a question raised in [3].

2.8. Of course there is an analogue of Theorem 2.3 for parafree groups in certain varieties, indeed in any Magnus variety containing $\mathfrak{S}_{2}$, the variety of all metabelian groups. In order to be able to carry over Theorem 2.3 to such varieties we need

LEMMA 2.1. Let $F$ be the free metabelian group freely generated by $a$ and $b$. If $u \in \gamma_{2} F$ then $F=\operatorname{gp}(a u, b)$ if and only if $u=\left[a, b^{i}\right]$ for some $i$.

Proof. Suppose $a u$ and $b$ generate $F$. Then, by hopficity, $a u$ and $b$ freely generate $F$. Let $\theta$ be the automorphism of $F$ defined by

$$
\theta: a \mapsto a u, b \mapsto b .
$$

Then $\theta$ is the identity modulo $\gamma_{2} F$. So by a theorem of Seymour Bachmuth [2] $\theta$ is inner. But the only elements of $F$ which centralize $b$ are the powers $b^{i}$ of $b$ (see e.g., [7]). Thus the lemma follows. 
We observe now that in a Magnus variety $\mathfrak{B}$ any set of elements which are independent modulo the derived group of a free group in $\mathfrak{B}$ freely generate a free $\mathfrak{B}$-group [6]. This remark, together with Lemma 2.1 allows us to carry Theorem 2.3 over, without any further comment, to

THEOREM 2.4. Let $\mathfrak{B}$ be any Magnus variety which contains the variety of all metabelian groups and let $\mathfrak{B}_{1}, \mathfrak{B}_{2}, \ldots$ be any countable family of proper subvarieties of $\mathfrak{B}$. Then there exists a parafree group $P$ of rank two in $\mathfrak{B}$ such that

(i) $P / V_{i}(P) \cong F / V_{i}(F)$ for $i=1,2, \ldots$, where $F$ is a free $\mathfrak{B}$-group of rank two;

(ii) $P$ is an ascending union of free $\mathfrak{B}$-groups of rank two;

(iii) $P$ is not a free $\mathfrak{B}$-group.

Notice that if $\mathfrak{S}_{2}$ is the variety of all metabelian groups then, by a theorem of D. E. Cohen [11] there are only a countably infinite number of subvarieties of $\mathfrak{S}_{2}$. So Theorem 2.4 provides us with a nonfree parafree group $P$ in the variety $\mathfrak{S}_{2}$ such that $P / U(P) \cong F / U(F)$ for every proper subvariety $\mathfrak{u}$ of $\mathfrak{S}_{2}$, where $F$ is a free $\varsigma_{2}$-group of rank two.

\section{Embedding theorems.}

3.1. Let $R$ be the free associative ring with unit element on $X$. Then $R$ may be viewed as a valuated ring (see P. M. Cohn [12]). Consequently $R$ carries with it a natural topology which makes $R$ into a topological ring. As such $R$ has a completion $\hat{R}$ (see again P. M. Cohn [12, pp. 453, 454]) which we denote by $(\hat{R}=) Z[[X]]$ (here $Z$ stands for the ring of integers). $\hat{R}$ is termed the power series ring on $X$. The elements $r$ in $\hat{R}$ may be thought of as formally infinite sums $r=\sum_{i=0}^{\infty} r_{i}$ where $r_{i}$, the homogeneous component of $r$ of degree $i$, is a finite sum of integer multiples of the monomials $x_{m_{1}} x_{m_{2}} \cdots x_{m_{l}}$ with $x_{m_{j}} \in X$ (and $r_{0} \in Z$ ). Put

$$
R_{i}=\left\{r \in \hat{R} \mid r_{0}=\cdots=r_{i-1}=0\right\}
$$

for $i=1, \ldots$ Then $R_{0}=\hat{R}$. Of course $R_{i}$ is an ideal of $R$ and the quotient rings $\hat{R} / R_{i}$ together with the natural homomorphisms from $\hat{R} / R_{i}$ to $\hat{R} / R_{j}(i \geqq j)$ constitute an inverse system of rings. The corresponding inverse limit (see e.g., Eilenberg and Steenrod [14])

$$
\bar{R}=\text { inlt } R / R_{n}
$$

is isomorphic to $\hat{R}$, essentially by definition.

Now let $F$ be the absolutely free group on a set $\left\{a_{x} \mid x \in X\right\}$ of generators indexed by $X$. Then again the system of groups $F / \gamma_{n} F$ together with the obvious homomorphisms constitute an inverse system. Let $\bar{F}$ be the inverse limit of this inverse system. Clearly every parafree group of rank $|X|$ can be embedded in $\bar{F}$. Moreover the mapping

$$
a_{x} \gamma_{n} F \mapsto 1+x+R_{n}
$$

defines a monomorphism $\phi_{n}$ of $F / \gamma_{n} F$ into $\hat{R} / R_{n}$ for every $n=1,2, \ldots$ (W. Magnus 
[23]). The functor inlt is left exact (see e.g., [14]). Therefore the monomorphisms $\phi_{n}$ define a monomorphism

$$
\text { inlt } \phi_{n}: \text { inlt } F / \gamma_{n} F \rightarrow \text { inlt } \hat{R} / R_{n} \text {. }
$$

So in particular every parafree group of rank $|X|$ can be embedded in $Z[[X]]$. Thus we have proved the

THEOREM 3.1. Every absolutely parafree group $P$ of rank $\mathfrak{n}$ can be embeddea in a power series ring on a set $X$ of $\mathfrak{n}$ generators. Moreover if $B$ is a parabasis for $P$ this embedding can be chosen so that $B$ maps onto $1+X=\{1+x \mid x \in X\}$.

3.2. We turn our attention now to the variety $\mathfrak{S}_{2}$ of all metabelian groups. Let $\Lambda$ be a well-ordered index set and let $R^{*}$ be the power-series ring in the generators $x_{i \lambda}(i=1,2, \lambda \in \Lambda)$ subject to the defining relations

$$
x_{2 \lambda} x_{1 \mu}=x_{1 \lambda} x_{1 \mu}=x_{2 \lambda} x_{2 \mu}-x_{2 \mu} x_{2 \lambda}=0 \quad(\lambda, \mu, \nu \in \Lambda) .
$$

It is easy to see that $R^{*}$ is a graded ring (the relations (1) an $\therefore$ omogeneous). Moreover every monomial $a(\neq 0)$ in the semigroup generated by the $x_{i \lambda}$ can be written uniquely in the form

$$
a=x_{i_{1}, \lambda_{1}} x_{i_{2}, \lambda_{2}} \cdots x_{i_{n}, \lambda_{n}} \quad\left(1 \leqq i_{j} \leqq 2, \lambda_{j} \in \Lambda\right)
$$

where

(i) if $i_{1}=1$ and $i_{2}=2$, then $i_{j}=2$ for $j \geqq 2$ and $\lambda_{2} \leqq \cdots \leqq \lambda_{n}$;

(ii) if $i_{1}=2$ then $i_{j}=2$ for $j \geqq 1$ and $\lambda_{1} \leqq \lambda_{2} \leqq \cdots \leqq \lambda_{n}$.

It follows that every element $r$ of $R^{*}$ can be written uniquely in the form $r=\sum_{i=0}^{\infty} r_{i}$ where $r_{n}$ is a finite sum of integer multiples of monomials of degree $n$. Now if we put

$$
R_{n}=\left\{r \in R^{*} \mid r_{0}=r_{1}=\cdots=r_{n-1}=0\right\},
$$

then $R_{n}$ is an ideal of $R^{*}$ and $\bigcap_{n=1}^{\infty} R_{n}=0$. It follows, as in 3.1 , that $\bar{R}=$ inlt $R^{*} / R_{n}$ may be identified with $R^{*}$. Moreover if $F$ is the free metabelian group (i.e., the free group in $\mathfrak{S}_{2}$ ) freely generated by $\left\{a_{\lambda} \mid \lambda \in \Lambda\right\}$, then for each $n$ the mapping

$$
\phi_{n}: a_{\lambda}+\gamma_{n} F \mapsto 1+x_{1, \lambda}+x_{2, \lambda}+R_{n}
$$

defines a monomorphism of $F / \gamma_{n} F$ into $R^{*} / R_{n}$ (this follows immediately by observing that the proofs of Theorem 1 and Theorem 2 in [8] are valid if the extra relations $x_{1 \lambda} x_{1 \mu}=0$ are added to the relations of the ring $R$ in [8]; indeed this is the content of an unpublished theorem of W. Magnus). So as before we have a monomorphism

$$
\text { inlt } \phi_{n}: \text { inlt } F / \gamma_{n} F \rightarrow \text { inlt } R^{*} / R_{n} \text {. }
$$

Since every parafree group in $\mathfrak{S}_{2}$ of rank $|X|$ is a subgroup of inlt $F / \gamma_{n} F$ we have proved the 
THEOREM 3.2. Every parafree group of rank $\mathfrak{n}$ in the variety of all metabelian groups can be embedded in a power series ring of the type defined by the relations (1) on $\mathfrak{n}$ generators.

Theorem 3.2 yields the

COROLLARY. Every parafree group in the variety of all metabelian groups can be embedded in a matrix group over a field.

In order to prove this corollary observe that it is enough to prove that every ring $R^{*}$ of the type described above can be embedded in a matrix ring over a field. In order to do so let $S$ be the quotient field of the ring of power-series in the commuting variables $a_{i \lambda}(i=1,2, \lambda \in \Lambda$, a well-ordered index set) over the field of rationals. Let $M_{2}(S)$ be the ring of $2 \times 2$ matrices over $S$. Put

$$
\bar{a}_{2 \lambda}=\left(\begin{array}{ll}
a_{2 \lambda} & 0 \\
0 & 0
\end{array}\right), \quad \bar{a}_{1 \lambda}=\left(\begin{array}{ll}
\theta & 0 \\
a_{1 \lambda} & 0
\end{array}\right) \quad(\lambda \in \Lambda) .
$$

Then

$$
\bar{a}_{2 \lambda} \bar{a}_{1 \mu}=\bar{a}_{1 \lambda} \bar{a}_{1 \mu}=\bar{a}_{2 \lambda} \bar{a}_{2 \mu}-\bar{a}_{2 \mu} \bar{a}_{2 \lambda}=0 \quad(\lambda, \mu \in \Lambda) .
$$

It follows easily from this observation that the mapping

$$
x_{i \lambda} \mapsto \bar{a}_{i \lambda}
$$

defines a monomorphism of the ring $R^{*}$ described above into $M_{2}(S)$ (cf. W. Magnus [24]). This completes the proof of the corollary.

3.3. We prove next

THEOREM 3.3. Every absolutely parafree group of countable rank can be embedded in an absolutely parafree group of rank two.

Theorem 3.3 may be viewed either as an analogue of a theorem of G. Higman, B. H. Neumann and Hanna Neumann [17] viz., every countable group can be embedded in a two-generator group or as a generalization of the corresponding statement for absolutely free groups.

We proceed now to the proof of Theorem 3.3. Thus let $F$ be the absolutely free group on $a$ and $b$ and let $P$ be a parafree group of countable rank. Since the free product of parafree groups is parafree (Theorem 1.3) we may assume that $P$ has a countably infinite parabasis

$$
X=\left\{\ldots, x_{-1}, x_{0}, x_{1}, \ldots\right\} .
$$

Put

$$
b_{i}=a^{-i} b a^{i} \quad(i=0, \pm 1, \ldots) .
$$

Then the $b_{i}$ 's freely generate a free subgroup $E$ of $F$. Since $X$ freely generates a free 
subgroup $B$ of $P$ (Theorem 4.1 in $\S 4$ ) we may form the generalized free product

$$
G=\{F * P ; E \stackrel{\oplus}{=} B\}
$$

amalgamating $E$ with $B$ according to the isomorphism

$$
\phi: b_{i} \mapsto x_{i} \quad(i=0, \pm 1, \ldots) .
$$

We claim that $G$ has the same lower central sequence as a free group of rank two. To see this observe first that modulo $\gamma_{2} F$ we have

$$
b_{i} \equiv b_{0} \quad \text { for all } i=0, \pm 1, \ldots
$$

Hence modulo $\gamma_{2} G$ it follows that

$$
x_{j} \equiv b_{j} \equiv b_{0} \quad \text { for all } j=0, \pm 1, \ldots
$$

But, modulo $\gamma_{2} P, X$ generates $P$. So, modulo $\gamma_{2} G, a$ and $b$ alone generate $G$. This means $G / \gamma_{n} G$ can be generated by two elements for every $n$.

Next we observe that $F / \gamma_{n} F$ is a quotient of $G$ for every $n \geqq 2$. To do so let us denote the natural homomorphism of $F$ onto $F / \gamma_{n} F$ by $\theta_{n}$. Now $P / \gamma_{n} P$ is a free nilpotent group of class $n-1$ freely generated by the images of the elements of $X$. Therefore the mapping

$$
\psi_{n}: x_{i} \gamma_{n} P \mapsto b_{i} \gamma_{n} F
$$

can be continued to a homomorphism, again denoted $\psi_{n}$, of $P / \gamma_{n} P$ into $F / \gamma_{n} F$. Let $\xi_{n}$ be the product of the natural homomorphism of $P$ onto $P / \gamma_{n} P$ and $\psi_{n}$. Then $\theta_{n}$ and $\xi_{n}$ agree on the amalgamated subgroup $E(=B)$. So they can simultaneously be extended to a homomorphism of $G$ onto $F / \gamma_{n} F$. But $G / \gamma_{n} G$ can be generated by two elements. So, by hopficity

$$
G / \gamma_{n} G \cong F / \gamma_{n} F
$$

Let us now put

$$
\gamma_{\omega} G=\bigcap_{n=1}^{\infty} \gamma_{n} G
$$

Then it follows from (1) that $G / \gamma_{\omega} G$ is parafree of rank two.

We complete the proof of Theorem 3.3 by proving that $P$ is naturally embedded in $G / \gamma_{\omega} G$ i.e.,

$$
P \cap \gamma_{\omega} G=1
$$

To this end let $a \in P(a \neq 1)$. Put

$$
P(k, l)=\gamma_{k} P \cdot P^{2^{l}}
$$

where $P^{r}$ is the subgroup generated by the $r$ th powers of the elements of $P$. Now for a suitable choice of $k$ and $l, a \notin P(k, l)$. 
$P / P(k, l)$ is a free group in an appropriate variety, say $\mathfrak{B}$, freely generated by the elements $x_{i} P(k, l)$. So for a suitable choice of the word $w$ we may write

$$
a P(k, l)=w\left(x_{0}, \ldots, x_{2^{m}-1}\right) P(k, l)
$$

(after rearranging our indexing if necessary).

$E(k, l)$ is, of course, a normal subgroup of the subgroup $F$ of $G$. We consider $F / E(k, l)$. Let $N / E(k, l)$ be the nor mal closure in $F / E(k, l)$ of $a^{2^{m}} E(k, l)$. Then $b_{0} N, \ldots, b_{2^{m}-1} N$ freely generate a free ${ }^{3}$-group $O / N$ and

$$
|F| N|O| N \mid=2^{m} \text {. }
$$

So $F / N$ is a finite 2-group. Of course it fol 'ws from our choice of $N$ that $w\left(b_{0}, \ldots, b_{2^{m}-1}\right) \notin N$.

Now let $\alpha$ be the natural homomorphism of $F$ nto $F / N$ and $\beta$ the natural homomorphism of $P$ onto $O / N$ defined by

$$
\beta: x_{i} \rightarrow b_{i}, N \quad(i=0, \pm 1, \ldots),
$$

where $i^{\prime}$ is the residue of $i$ modulo $2^{m}$ lying in the set $\left\{0,1, \ldots, 2^{m}-1\right\}$. Then $\alpha$ and $\beta$ agree whenever they both are defined. So they can simuitaneously be extended to a homomorphism $\gamma$ of $G$ onto $F / N$. We have arranged matters so that

$$
a \gamma=w\left(b_{0}, \ldots, b_{2^{m}-1}\right) N \neq 1 .
$$

If $c$ is the class of $F / N$ this implies that $a \notin \gamma_{c+1} G$. It follows immediately therefore that (2) holds. This completes the proof of Theorem 3.3.

Whether $G$ itself (above) is parafree is unclear, but it seems likely.

3.4. There is no analogue for Theorem 3.3 for soluble varieties. Thus for example a parafree subgroup of a parafree group of rank $\mathfrak{n}$ in the variety of all metabelian groups is of rank at most $\mathfrak{n}$. This follows without much difficulty from the results of $\S 4$ and Theorem 42.55 in [29].

\section{Subgroup theorems.}

4.1. We begin our subgroup theorems with

THEOREM 4.1. Let $P$ be a parafree group in a Magnus variety $\mathfrak{M}$ and let $X$ be a subset of $P$ which freely generates modulo $\gamma_{2} P$ a free abelian group. Then $X$ freely generates a free $\mathfrak{M}$-group.

Proof. Put $H=\operatorname{gp}(X)$. Now $P / \gamma_{c} P$ is a free group in an appropriate variety $\mathfrak{B}_{c}$. Since $P / \gamma_{c} P$ is a torsion-free nilpotent group it follows that it is residually a finite $p$-group for every prime $p$ (see [15]). So by [6, Theorem 1], $X$ freely generates, modulo $\gamma_{c} P$, a free group in $\mathfrak{B}_{c}$. Therefore $X$ freely generates a free $\mathfrak{M}$-group (see the introductory remarks in [3]).

One might conjecture that in any absolutely parafree group $P$ if $H=\mathrm{gp}(X)$ is a subgroup of $P$ such that $X$ freely generates $H$ modulo $\gamma_{2} H$, then $H$ is freely 
generated by $X$. This is false, however. To see this let

$$
P=\left(a, b, c ; a^{2} b^{2} c^{-3}\right) \text {. }
$$

Then $P$ is parafree. For by [9] $P$ is residually nilpotent. Moreover it follows from the arguments in [3] that $P$ has the same lower central sequence as an absolutely free group. Let

$$
Q=\operatorname{gp}\left(a, b, c^{-1} a c, c^{-1} b c\right) .
$$

Then $Q$ is defined by the single relation

$$
a^{2} b^{2}=\left(c^{-1} a c\right)^{2}\left(c^{-1} b c\right)^{2} .
$$

So $Q$ contains as a subgroup of index 2 the group (see [32])

$$
R=\left(x_{1}, y_{1}, x_{2}, y_{2}, x_{3}, y_{3} ; x_{1}^{-1} y_{1}^{-1} x_{1} y_{1} x_{2}^{-1} y_{2}^{-1} x_{2} y_{2} x_{3}^{-1} y_{3}^{-1} x_{3} y_{3}\right) \text {. }
$$

But now $x_{1}, x_{2}, x_{3}, y_{1}, y_{2}, y_{3}$ freely generate a free abelian group modulo $\gamma_{2} R$. However $R$ is not free (nor parafree).

4.2. We prove next

THEOREM 4.2. The abelian subgroups of an absolutely parafree group are free cyclic whereas the nonabelian two-generator subgroups are absolutely free of rank two.

We begin the proof of Theorem 4.2 by proving the latter statement first. Thus let $P$ be parafree and suppose $Q=\mathrm{gp}(u, v)$ is a two-generator subgroup of $P$. Assume $Q$ is nonabelian. Then by Theorem 3.1 we may assume $u$ and $v$ are noncommuting elements of $\hat{R}=Z[[X]]$ of the form

$$
u=\sum_{i=0}^{\infty} u_{i}, \quad v=\sum_{i=0}^{\infty} v_{i}
$$

where $u_{0}=1=v_{0}$. Let $\tilde{R}$ be the closed subring generated by $u$ and $v$ in $\hat{R}$. If $\tilde{R}$ is actually the power-series ring on $u-1$ and $v-1$ then it follows from Magnus' theorem [23] that $u$ and $v$ freely generate an absolutely free group. So it is enough to prove the

Proposition 4.1. Let $a$ and $b$ be noncommuting elements of $\hat{R}=Z[[X]]$ both of whose constant terms are zero. Then the closed subring generated by $a$ and $b$ is the power-series on $a$ and $b$ over $Z$.

Proposition 4.1 is due to P. M. Cohn. It supersedes my original result which is at the same time both weaker and stronger than Proposition 4.1.

Proof of Proposition 4.1. The proof is by induction on the order $\nu(a b-b a)$ of $a b-b a$ where $\nu(x)=n$ if $x \in R_{n}$ and $x \notin R_{n+1}$ with $\nu(0)=\infty$. If the closed subring generated by $a$ and $b$ is not the power-series ring on $a$ and $b$ over $Z$, they satisfy a relation of the form

$$
n+a f+b g=0
$$


where $f$ and $g$ are power-series in $a$ and $b$ and $n$ is an integer. Since both $a$ and $b$ have constant term $0, n=0$. So (1) takes on the form $a f+b g=0$. So, assuming that $\nu(a) \geqq v(b)$ there exists, by Theorem 4.1 of [12], $c \in \hat{R}$ such that

$$
a=b c \quad(\nu(c)<\nu(a)) .
$$

Since $a b-b a \neq 0, c b-b c \neq 0$. Moreover

$$
\nu(c b-b c)<\nu(a b-b a) .
$$

So inductively the closed subring generated by $c$ and $b$ is the power-series ring over $Z$ on $c$ and $b$. But then a straightforward argument shows that the closed subring generated by $(a=) b c$ and $b$ is likewise the power-series $\operatorname{ring}$ over $Z$ on $a$ and $b$. This proves the proposition and with it one part of Theorem 4.2.

To prove the first part of Theorem 4.2 one proceeds as follows. Let $L$ be an unrestricted free lie algebra over the rationals (see e.g., [25, p. 302]). Then $L$ can be turned into a group $L^{\times}$by introducing a binary operation $\times$into $L$ by means of the Baker-Campbell-Hausdorff formula (see e.g., [19, p. 174]). Now it follows from results of Lazard (see the remarks after Theorem 1.9, p. 151 in [22]) that an absolutely parafree group can always be embedded in such a group $L^{\times}$. But as W. Magnus has shown (see [25]) the two-generator abelian subgroup of $L^{\times}$are free cyclic (the proof in [25] that the two-generator subgroups of $L^{\times}$are free seems incomplete; the abelian version however is certainly complete). Hence the abelian subgroups of an absolutely parafree group are locally cyclic. But the abelian subgroups of free nilpotent groups are free abelian (E. Witt [34]). Hence it follows that the abelian subgroups of an absolutely parafree group are residually-infinitecyclic. Now a locally cyclic residually infinite-cyclic group is clearly, free cyclic. So it follows that the abelian subgroups of a parafree group are free cyclic. This completes the proof of Theorem 4.2.

4.3. We turn our attention now to the variety of all metabelian groups. Our aim is to prove

THEOREM 4.3. Let $P$ be parafree in the variety of all metabelian groups. Then the two-generator subgroups of $P$ are isomorphic to two-generator subgroups of free metabelian groups.

We observe that $P$ may be viewed as a subgroup of the ring $R^{*}$ described in 3.2. $R^{*}$ is a graded ring and $P$ clearly maps onto a torsion-free subgroup of $R^{*}$. So every finitely generated abelian subgroup of $P$ is free abelian.

Consequently the proof of Theorem 4.3 reduces to a consideration of the twogenerator nonabelian subgroups of $P$. Thus let $T=\mathrm{gp}(t, u)$ be a nonabelian subgroup of $P$. If $t$ and $u$ are independent modulo $\gamma_{2} P$ then, by Theorem 4.1, $T$ is a free metabelian group freely generated by $t$ and $u$.

We are left therefore with the case where $T$ is infinite cyclic modulo $\gamma_{2} P$. First however we would like to point out that the nonabelian two-generator subgroups 
of a free metabelian group (indeed of a free soluble group) are well known. (Curiously enough very little is known about the three-generator subgroups.) In fact it follows easily on putting e.g., Theorem 2 of [6] and Corollary 3 of [7] together that a nonabelian two-generator subgroup of a free metabelian group is either

(a) free metabelian of rank two, or

(b) the (standard) wreath product of one infinite cyclic group by another.

Our objective now is to show that if $T=\mathrm{gp}(t, u)$ is a nonabelian subgroup of $P$ such that $T$ is infinite cyclic modulo $\gamma_{2} P$ then $T$ is given by (b). Indeed by Euclid's algorithm we may assume $u \in \gamma_{2} P$. So we have only to prove that the conjugates $\ldots, t u t^{-1}, u, t^{-1} u t, \ldots$ of $u$ by the powers of $t$ freely generate a free abelian group.

We repeat that we may think of $P$ as a subgroup of the graded ring $R^{*}$ given in 3.2. Moreover we may assume that the elements

$$
1+x_{\lambda}\left(=1+x_{1 \lambda}+x_{2 \lambda}\right) \quad(\lambda \in \Lambda)
$$

constitute a parabasis for $P$ and also that $\Lambda$ is finite (see the proof of Theorem 5.1).

Notice that every element $u$ in $P$ may be expressed in the form

$$
u=1+f+x_{1, \lambda_{1}} f_{1}+\cdots+x_{1, \lambda_{n}} f_{n} \quad\left(\lambda_{1}<\lambda_{2}<\cdots<\lambda_{n}\right)
$$

where $f, f_{1}, \ldots, f_{n}$ are power series in the ("commuting variables") $x_{2 \lambda}$ with integral coefficients. If $u \in \gamma_{2} P$ then $f=0$; for otherwise on adding the relations $x_{1, \lambda}=0(\lambda \in \Lambda)$ to $R^{*}$ the image of $P$ in the resultant quotient ring is abelian and the image of $u \neq 1$. So if $u \in \gamma_{2} P$ the expression (1) becomes

$$
u=1+x_{1, \lambda_{1}} f_{1}+\cdots+x_{1, \lambda_{n}} f_{n} \quad\left(\lambda_{1}<\lambda_{2}<\cdots<\lambda_{n}\right) .
$$

It follows either from the defining relations of $R^{*}$ or (perhaps more easily) from the faithful representation of $R^{*}$ in the matrix $\operatorname{ring} M_{2}(S)$ described towards the end of $\S 3.2$, that the expression (2) is unique in the sense that $u=1$ if and only if $f_{1}=f_{2}=\cdots=f_{n}=0$.

Our objective is to prove that if $u \neq 1$ then the conjugates of $u$ by the powers of $t$ freely generate a free abelian group. Since $t \notin \gamma_{2} P$ we may express $t$ in the form

$$
t=\left(1+x_{\mu_{1}}\right)^{\tau} \cdots\left(1+x_{\mu_{i k}}\right)^{\tau} t^{\prime} \quad\left(t^{\prime} \in \gamma_{2} P, \tau_{1} \tau_{2} \cdots \tau_{k} \neq 0\right) .
$$

Now $\gamma_{2} P$ is abelian. So conjugation of $u$ by $t$ is equivalent to conjugation by $t t^{\prime-1}$. Moreover, since $u$ commutes with every $x_{1, \lambda}$ and $1+x_{\mu}=\left(1+x_{2, \mu}\right)\left(1+x_{1, \mu}\right)$, conjugation of $u$ by $t$ is equivalent to conjugation by

$$
t^{*}=\left(1+x_{2, \mu_{1}}\right)^{\tau_{1}} \cdots\left(1+x_{2, \mu_{k}}\right)^{\tau_{k}} .
$$

Thus if $u$ is given by (1) it follows that for every pair of integers $e$ and $m$

$$
\begin{aligned}
\left(u^{e}\right)^{t^{m}=\left(u^{e}\right)^{t^{* m}}=1}+x_{1, \lambda_{1}} f_{1} e\left(\left(1+x_{2, \mu_{1}}\right)^{\tau_{1}} \cdots\left(1+x_{2, \mu_{k}}\right)^{\tau_{k}}\right)^{m}+\cdots \\
+x_{1, \lambda_{n}} f_{n} e\left(\left(1+x_{2, \mu_{1}}\right)^{\tau_{1}} \cdots\left(1+x_{2, \mu_{k}}\right)^{\tau_{k}}\right)^{m} .
\end{aligned}
$$


Now suppose that $u \neq 1$, that $m_{1}, \ldots, m_{l}$ are distinct integers, that $e_{1}, \ldots, e_{l}$ are nonzero integers and that

$$
p=\left(u^{e_{1}}\right)^{t^{m_{1}}} \cdots\left(u^{e_{l}}\right)^{t^{m_{l}}}
$$

Then

$$
\begin{gathered}
p=1+x_{1, \lambda_{1}} f_{1}\left\{e_{1}\left(\left(1+x_{2, \mu_{1}}\right)^{\tau_{1}} \cdots\left(1+x_{2, \mu_{k}}\right)^{\tau_{k}}\right)^{m_{1}}+\cdots+e_{l}\left(\left(1+x_{2, \mu_{1}}\right)^{\tau_{1}} \cdots\left(1+x_{2, \mu_{k}}\right)^{\xi_{k}}\right)^{m_{\imath}}\right\} \\
+\cdots+x_{1, \lambda_{n}} f_{n}\left\{e_{1}\left(\left(1+x_{2, \mu_{1}}\right)^{\tau_{1}} \cdots\left(1+x_{2, \mu_{k}}\right)^{\tau_{k}}\right)^{m_{1}}+\cdots\right. \\
\left.+e_{l}\left(\left(1+x_{2, \mu_{1}}\right)^{\tau_{1}} \cdots\left(1+x_{2, \mu_{k}}\right)^{\tau_{k}}\right)^{m_{\imath}}\right\} .
\end{gathered}
$$

So by the uniqueness of the expression (2) $p=1$ if and only if

$$
f_{i}\left\{\sum_{j=1}^{l} e_{j}\left(\left(1+x_{2, \mu_{1}}\right)^{\tau_{1}} \cdots\left(1+x_{2, \mu_{k}}\right)^{\tau_{k}}\right)^{m_{j}}\right\}=0
$$

for $i=1, \ldots, n$. Since $u \neq 1, f_{i} \neq 0$ for some $i$. Now the ring of power series in the $x_{2, \lambda}$ over the integers has no zero-divisors. Therefore if

$$
\sum_{j=1}^{l} e_{j}\left(\left(1+x_{2, \mu_{1}}\right)^{\tau_{1}} \cdots\left(1+x_{2, \mu_{h}}\right)^{\tau_{k}}\right)^{m_{j}} \neq 0,
$$

$p \neq 1$. On post-multiplying by suitable powers of the $1+x_{2, \mu_{i}}$ we may assume that the exponents $\tau_{i}$ in (3) are all positive. But then, remembering that $e_{1}, \ldots, e_{l}$ are all nonzero and that the integers $m_{1}, \ldots, m_{l}$ are distinct, the result follows readily on comparing monomials of the highest degree occurring in (3). This completes the proof of Theorem 4.3.

\section{Residual properties.}

5.1. We start out with the proof of

THEOREM 5.1. A parafree group is residually parafree-of-finite-rank.

Proof. Let $P$ be a parafree group in a variety $\mathfrak{B}=\mathfrak{B}^{\circ}$ with parabasis $X$ (for the definition of $\mathfrak{B}^{\circ}$ see $\left.\S 0.6\right)$. Now suppose $a \in P(a \neq 1)$. Then $a \notin \gamma_{n} P$ for some $n$. Thus we can find $x_{1}, \ldots, x_{k} \in X$ such that

$$
a \gamma_{n} P=w\left(x_{1}, \ldots, x_{k}\right) \gamma_{n} P .
$$

We now add to the relations of $P$ the relations

$$
x=1 \text { if } x \in X, x \notin\left\{x_{1}, \ldots, x_{k}\right\}
$$

together with the minimal number of relations which will ensure residual nilpotence of the appropriate factor group of $P$. More precisely let

$$
K=\operatorname{gp}_{P}\left(x \mid x \in X \backslash\left\{x_{1}, \ldots, x_{k}\right\}\right)
$$

and define $J$ by

$$
J / K=\gamma_{\omega}(P / K) .
$$

Then $P / J$ is the required parafree image of $P$ of finite rank in which $a$ does not have 
a trivial image. To see this notice that $P / K / \gamma_{n}(P / K)$ is the free nilpotent group in $\mathfrak{B}$ of class $n-1$ freely generated by the images of $x_{1} K, \ldots, x_{k} K$ modulo $\gamma_{n}(P / K)$. So $w\left(x_{1} K, \ldots, x_{k} K\right)$ is not the identity modulo $\gamma_{n}(P / K)$ i.e., $a$ has a nontrivial image in $P / K / \gamma_{n}(P / K)$. Clearly $J \leqq\left(\gamma_{n} P\right) K$. So $a \notin J$.

It remains to prove only that $P / J$ is parafree in $\mathfrak{B}$ of finite rank. To see this observe that we have already shown that $P / J / \gamma_{n}(P / J)$ is a free nilpotent group in $\mathfrak{B}$ of class $n-1$ and rank $k$. Since $P / J$ is clearly residually nilpotent we have proved that $P / J$ is parafree in $\mathfrak{B}$ of finite rank.

There remains the possibility that parafree groups are residually-finitelygenerated-parafree. This is not the case however. For example if we consider absolutely parafree groups let us take $P$ to be a parafree group obtained as a properly ascending union of free groups $F_{i}$ of rank two (cf. §3):

$$
P=\bigcup_{i=1}^{\infty} F_{i} \quad\left(F_{i} \leqq F_{i+1}, F_{i} \neq F_{i+1}\right) .
$$

Suppose, if possible, that $P / N$ is a finitely generated nonabelian parafree quotient of $P$. Then

$$
P / N=\bigcup_{i=1}^{\infty} F_{i} N / N
$$

Since $P / N$ is finitely generated, ultimately $F_{i} N / N$ coincides with all the $F_{j} N / N$ for $j \geqq i$ if $i$ is chosen large enough. So $F_{i} N / N$ is a nonabelian absolutely parafree group on two generators. Hence (for example by Theorem 4.2) $F_{i} N / N$ is free of rank two. By hopficity this implies $F_{i} \cap N=1$ since $F_{i} N / N \cong F_{i} / F_{i} \cap N$. Similarly

$$
F_{j} \cap N=1 \text { for all } j \geqq i \text {. }
$$

But $N \subseteq \bigcup_{j=i}^{\infty} F_{j}$. So $N=1$. This contradicts the choice of $N$. Hence $P$ has no finitely generated nonabelian parafree images. So $P$ is certainly not residually finitely-generated-parafree.

5.2. For absolutely parafree groups Theorem 5.1 can be improved somewhat. Indeed we shall prove

THEOREM 5.2. A noncyclic absolutely parafree group is residually a parafree group of rank two.

Let the nonabelian group $P$ be absolutely parafree. We have to prove that $P$ is residually parafree of rank two. Since $P$ is, by Theorem 5.1, residually parafree of finite rank we may assume $P$ is of finite rank $n+1$. We shall prove that if $n \geqq 2$ then $P$ is residually parafree of rank $n$, thereby proving Theorem 5.2.

Let $X=\left\{x_{1}, \ldots, x_{n+1}\right\}$ be a parabasis for $P$ and let $\hat{R}$ be the power series ring over $Z$ in $\xi_{1}, \ldots, \xi_{n+1}$. Then, by Theorem 3.1, we may assume that $P$ is a subgroup of $\hat{R}$ with

$$
x_{i}=\xi_{i}+1 \quad(i=1, \ldots, n+1) .
$$


Now let $a \in P(a \neq 1)$. Then

$$
a=\sum_{i=0}^{\infty} a_{i} \quad\left(a_{0}=1\right) .
$$

Now each $a_{i} \neq 0$ can be expressed uniquely as a finite sum of integer multiples of monomials of degree $i$ :

$$
a_{i}=\sum c_{j_{1}, j_{2}, \ldots, j_{i}} \xi_{j_{1}} \xi_{j_{2}} \cdots \xi_{j_{t}} \quad\left(c_{j_{1}, \ldots, j_{i}} \in Z \mid 0\right) .
$$

We shall produce an endomorphism $\phi$ of $\hat{R}$ with the following properties

(i) $a \phi \neq 1$ and

(ii) $1 \phi=1, \xi_{1} \phi=\xi_{1}, \ldots, \xi_{n} \phi=\xi_{n}, \xi_{n+1} \phi=u-1$ where $u \in \operatorname{gp}\left(1+\xi_{1}, \ldots, 1+\xi_{n}\right)$.

It is convenient to divide the proof of the existence of such an endomorphism $\phi$ into two cases.

Case 1. There exists a component $a_{i} \neq 0$ of $a(i \geqq 1)$ in which some monomial does not contain $\xi_{n+1}$.

Let $a_{r} \neq 0$ be the first component of $a(r \geqq 1)$ with a monomial which does not contain $\xi_{n+1}$. Let $\alpha_{1}, \alpha_{2}, \ldots, \alpha_{k}$ be all the monomials in $a_{r}$ which do not contain $\xi_{n+1}$.

Consider the $(r+1)$-fold commutator

$$
\left[1+\xi_{1}, 1+\xi_{2}, \ldots, 1+\xi_{2}\right]=1+\xi \quad \text { (say), }
$$

and define an endomorphism $\phi$ of $\hat{R}$ by

$$
\phi: \xi_{1} \mapsto \xi_{1}, \ldots, \xi_{n} \mapsto \xi_{n}, \xi_{n+1} \mapsto \xi, 1 \mapsto 1 .
$$

Observe now that the first nonzero homogeneous component of positive degree of $a \phi$ is $m_{1} \alpha_{1}+\cdots+m_{k} \alpha_{k}$ where $m_{i}$ is the coefficient of $\alpha_{i}$ in $a_{r}$. So $a \phi \neq 1$. This establishes the existence of $\phi$ in this case.

Case 2. For every $i \geqq 1$ for which $a_{i} \neq 0$, every monomial occurring in $a_{i}$ contains $\xi_{n+1}$.

Let $a_{r} \neq 0$ be the first component of $a(r \geqq 1)$ which contains a monomial $\alpha$ in which $\xi_{n+1}$ occurs no more often than in any other monomial occurring in the components of $a$ of positive degree. We construct first a homomorphism $\theta$ of $\hat{R}$ into $\hat{R}$ which enables us to assume that $a_{r}$ is actually the first nonzero component of $a(r \geqq 1)$. Let us therefore compute the $(l+1)$-fold commutator

$$
\left[1+\xi_{1}, 1+\xi_{n+1}, \ldots, 1+\xi_{n+1}\right]=1+\xi_{1} \xi_{n+1}^{l}+\cdots \quad(=1+v) .
$$

If we order monomials in $\xi_{1}, \ldots, \xi_{n+1}$ first by length and then lexicographically, the dots in (1) signify later monomials than $\xi_{1} \xi_{n+1}^{l}$.

Now let $\theta$ be the endomorphism of $\hat{R}$ defined by

$$
\theta: \xi_{1} \mapsto \xi_{1}, \ldots, \xi_{n} \mapsto \xi_{n}, \xi_{n+1} \mapsto v, 1 \mapsto 1 .
$$

Of course $\theta$ depends on $l$. Let $\alpha=\alpha_{1}, \alpha_{2}, \ldots, \alpha_{k}$ be the monomials in $a_{r}$ which 
contain $\xi_{n+1}$ with the same multiplicity $m$ as $\alpha$. Now if $a_{i} \neq 0(1 \leqq i<r)$ then $\xi_{n+1}$ occurs in every monomial in $a_{i}$ with a multiplicity greater than $m$. So by choosing $l$ sufficiently large we can ensure that the order of the image of each monomial in every $a_{i} \neq 0(i \neq 1, i \neq r)$ under $\theta$ is greater than the order of $\alpha_{i} \theta(1 \leqq i \leqq k)$. It follows from this remark that $a \theta=1+m_{1} \alpha_{1} \theta+\cdots+m_{k} \alpha_{k} \theta+$ terms of higher degree than the order of $\alpha_{1} \theta, \ldots, \alpha_{k} \theta$, where $m_{i}$ is the coefficient of $\alpha_{i}$ occurring in $a_{r}$. Notice that $\alpha_{1} \theta, \alpha_{2} \theta, \ldots, \alpha_{k} \theta$ are all of the same order. We may suppose that $\alpha_{1}$ is earliest in the lexicographic ordering among $\alpha_{1}, \alpha_{2}, \ldots, \alpha_{k}$. It follows from (1) that the earliest monomial, say $\mu$, occurring in $\alpha_{1} \theta, \alpha_{2} \theta, \ldots, \alpha_{k} \theta$ arises from $\alpha_{1}$ on substituting $\xi_{1} \xi_{n+1}^{l}$ in place of $\xi_{n+1}$. Moreover this is the only time $\mu$ arises. So $a \theta=1+\mu+\cdots$ where again the dots signify the possible presence of later monomials. So $a \theta \neq 1$.

We have shown that we may assume $a_{r}$ is the first nonzero component of $a$ $(r \geqq 1)$. Notice that every monomial $(\neq 1)$ in the components $(\neq 0)$ of $a$ involve $\xi_{n+1}$ and that there are monomials in the first nonzero homogeneous component $a_{r}(r \geqq 1)$ in which $\xi_{n+1}$ occurs with minimal multiplicity $m$. Indeed let $\alpha_{1}, \alpha_{2}, \ldots$, $\alpha_{k}$ be the monomials of $a_{r}$ in which $\xi_{n+1}$ occurs with multiplicity $m$. Again we suppose that the $\alpha_{i}$ are ordered lexicographically and that $\alpha_{1}$ is first in the ordering.

Now observe that

$$
\left[1+\xi_{1}, 1+\xi_{n}\right]=1+\xi_{1} \xi_{n}+\cdots=1+u
$$

We define an endomorphism $\phi$ of $\hat{R}$ as follows:

$$
\phi: \xi_{1} \mapsto \xi_{1}, \ldots, \xi_{n} \mapsto \xi_{n}, \xi_{n+1} \mapsto u, 1 \mapsto 1
$$

Then the first monomial in $\alpha_{1} \phi$ is earlier than any other monomial $(\neq 1)$ that arises in computing $a \phi$. Actually we have only to check that if we substitute $\xi_{1} \xi_{n}$ for $\xi_{n+1}$ in $\alpha_{1}, \alpha_{2}, \ldots, \alpha_{k}$ then of the resultant monomials the one arising from $\alpha_{1}$ is earliest in the ordering. This completes the proof of the existence of $\phi$.

To see how the existence of the endomorphism $\phi$ is relevant to parafreeness consider $P \phi$. Notice that modulo $\gamma_{2} P, P$ is generated by $1+\xi_{1}, \ldots, 1+\xi_{n+1}$. So $P \phi$ is generated modulo $\gamma_{2}(P \phi)$ by $1+\xi_{1}, \ldots, 1+\xi_{n}$. Hence $P \phi / \gamma_{t}(P \phi)$ is an $n$-generator group for every $t \geqq 2$.

But observe that $\hat{R}$ maps onto $\hat{S}=Z\left[\left[\xi_{1}, \ldots, \xi_{n}\right]\right]$. Now if $S_{t}$ is the ideal of $\hat{S}$ consisting of elements of order at least $t$ then $1+\xi_{1}, \ldots, 1+\xi_{n}$ freely generate modulo $S_{t}$ a free nilpotent group of class $t-1$. So $P \phi / \gamma_{t+1}(P \phi)$ is a free nilpotent group of rank $n$ and class $t$ for every $t \geqq 2$. Since $P \phi$ is obviously residually nilpotent $P \phi$ is parafree. This completes the proof of Theorem 5.2.

In much the same way, keeping in mind that a free Lie ring can always be embedded in a free associative ring (see e.g., [19]), one can prove 
Similarly one has (see Clifford and Preston [10] for a discussion of semigroups)

Noncyclic free semigroups are residually free of rank two.

5.3. We prove finally the analogue of Theorem 5.2 for parafree groups in the variety of all metabelian groups.

THEOREM 5.3. A noncyclic parafree group $P$ in the variety of all metabelian groups is residually a parafree group of rank two.

We may assume, by Theorem 5.1 , that $P$ is of finite rank $n(n \geqq 2)$.

Now suppose $a \in P(a \neq 1)$. Then $a \notin \gamma_{c} P$ for some $c \geqq 2$. $P / \gamma_{c} P$ is of course free in the obvious variety, $\mathfrak{B}$ say. By a theorem of Hanna Neumann [29] there is a normal subgroup $K$ of $P$ containing $\gamma_{c} P$ such that $a \notin K$ and

$$
P / \gamma_{c} P \mid K / \gamma_{c} P \text { is free of rank } 2 \text { in } \mathfrak{B} \text {. }
$$

Since free groups in $\mathfrak{B}$ are projective (see [29]) $K / \gamma_{c} P$ is complemented in $P / \gamma_{c} P$ by $L / \gamma_{c} P$ (say):

$$
P / \gamma_{c} P=\left(K / \gamma_{c} P\right) \cdot\left(L / \gamma_{c} P\right), \quad L / \gamma_{c} P \cap K / \gamma_{c} P=1 .
$$

So computing the factor derived group of $P / \gamma_{c} P$ we find

$$
P / \gamma_{2} P \cong\left(K / \gamma_{2} K[K, L] \gamma_{c} P\right) \times L / \gamma_{2} L \cdot \gamma_{c} P \text {. }
$$

The extreme right-hand side is free abelian of rank two, the left-hand side is free abelian of rank $n$. Hence $K /\left(\gamma_{2} K\right)[K, L]\left(\gamma_{c} P\right)$ is free abelian of rank $n-2$. Choose now elements

$$
a_{1}, \ldots, a_{n-2} \in K, \quad a_{n-1}, a_{n} \in L
$$

so that $a_{1}, a_{2}, \ldots, a_{n-2}$ freely generate $K$ modulo $\left(\gamma_{2} K\right)[K, L]\left(\gamma_{c} P\right)$ and $a_{n-1}, a_{n}$ freely generate $L$ modulo $\left(\gamma_{2} L\right)\left(\gamma_{c} P\right)$. By hopficity it follows that $a_{1}, a_{2}, \ldots, a_{n}$ freely generate $P$ modulo $\gamma_{2} P$. Moreover again by hopficity we find that

$$
K=\operatorname{gp}_{P}\left(a_{1}, \ldots, a_{n-2}\right)\left(\gamma_{c} P\right) .
$$

We are now in position to complete the proof of Theorem 5.3. Thus we define

$$
I=\operatorname{gp}_{P}\left(a_{1}, \ldots, a_{n-2}\right)
$$

and $J$ by

$$
J / I=\gamma_{\omega}(P / I) .
$$

Then we claim $P / J$ is parafree of rank two in the variety of all metabelian groups and that $a \notin J$. It is of course clear that $a \notin J$ since $a \notin K$.

To see that $P / J$ is parafree we observe first that, by its very definition $P / J$ is residually nilpotent. Moreover $a_{1}, a_{2}, \ldots, a_{n}$ freely generate $P$ modulo $\gamma_{c} P$ for every $c \geqq 1$. Hence $P / I\left(\gamma_{c} P\right)$ is free, in the appropriate variety, of rank two. But $J$ is the least normal subgroup of $P$ containing $I$ such that $P / J$ is residually nilpotent. 
Hence $J \leqq I\left(\gamma_{c} P\right)$. Therefore $P / J / \gamma_{c}(P / J)$ has a free nilpotent group $P / I\left(\gamma_{c} P\right)$ of rank two as factor group. But $P / J\left(\gamma_{c} P\right)$ is itself a two-generator group since $a_{1}, a_{2}, \ldots, a_{n}$ generate $P$ modulo $\gamma_{c} P$. This completes the proof of Theorem 5.3.

Finally we have

THEOREM 5.4. Let $\mathfrak{B}$ be a variety of groups and suppose $\mathfrak{B}$ is the union of its subvarieties $\mathfrak{B}_{\lambda}(\lambda \in \Lambda)$. Then a parafree group $P$ in $\mathfrak{B}$ is residually parafree in $\left\{\mathfrak{B}_{\lambda} \mid \lambda \in \Lambda\right\}$.

Proof. Let $a \in P(a \neq 1)$. Then $a \notin \gamma_{n} P$ for some $n$. Since $\mathfrak{B}$ is the union of the subvarieties $\mathfrak{B}_{\lambda}$ there exists $\lambda \in \Lambda$ such that

$$
a \gamma_{n} P \notin V_{\lambda}\left(P / \gamma_{n} P\right) .
$$

Hence if we define $V_{\lambda}^{\circ}(P)$ by

$$
V_{\lambda}^{\circ}(P) / V_{\lambda}(P)=\gamma_{\omega}\left(P / V_{\lambda}(P)\right),
$$

then $a \notin V_{\lambda}^{\circ}(P)$. We claim that $P / V_{\lambda}^{\circ}(P)$ is parafree in $\mathfrak{B}_{\lambda}$. This is clearly the case since $P / V_{\lambda}^{\circ}(P)$ is residually nilpotent by definition and

$$
P / V_{\lambda}^{\circ}(P) / \gamma_{n}\left(P / V_{\lambda}^{\circ}(P)\right) \cong P / \gamma_{n} P / V_{\lambda}^{\circ}\left(P / \gamma_{n} P\right)
$$

This completes the proof.

6. Parafree groups in product varieties.

6.1. We begin by proving

THEOREM 6.1. Let $\mathfrak{B}$ be any variety of groups. Then the parafree groups in $\mathfrak{A} \mathfrak{B}$ are torsion-free.

The first step in the proof of Theorem 6.1 is the following

LEMMA 1. Let $G$ be a group with an abelian normal subgroup $H$ such that $G / H$ is a p-group. Then for $n \geqq 2$

$$
\gamma_{n} G / \gamma_{n+1} G \text { is a p-group. }
$$

Proof. We prove first that $\gamma_{2} G / \gamma_{3} G$ is a p-group. Thus let $x, y \in G$. Then we have to show that, modulo $\gamma_{3} G,[x, y]$ is of order a power of $p$. But $x^{p^{r}} \in H$ and $y^{p^{r}} \in H$ for some choice of $r \geqq 1$. So

$$
1=\left[x^{p^{r}}, y^{p^{r}}\right] \equiv[x, y]^{p^{2 r}} \text { modulo } \gamma_{3} G \text {. }
$$

The rest of the lemma follows easily by induction.

The most important step in the proof of Theorem 6.1 is

LEMMA 2. A parafree group $P$ in $\mathfrak{A} \mathfrak{B}$, where $\mathfrak{B}$ is a variety of locally finite p-groups, is torsion-free. 
Proof. By Theorem 5.1 we may assume $P$ is of finite rank $n$. By Lemma $1, P$ is residually a finite $p$-group. So $V(P)$ is an abelian group which is residually a finite $p$-group. Therefore there are no elements of order prime to $p$ in $V(P)$.

Now let $F$ be free in $\mathfrak{A} \mathfrak{B}$ of rank $n$. Then since $n<\infty F / V(F)^{p^{i}}$ is nilpotent. Hence

$$
F / V(F)^{p^{i}} \cong P / V(P)^{p^{i}}
$$

This isomorphism provides us with an isomorphism

$$
V(F) / V(F)^{p^{i}} \cong V(P) / V(P)^{p^{i}} \quad(i=1,2, \ldots) .
$$

Now $V(F)$ is free abelian of finite rank. So $V(F) / V(F)^{p^{1}}$ is a direct product of (a finite number of) cyclic groups of order $p^{i}$.

On the other hand consider the abelian group $V(P) . V(P)$ is reduced. So if $V(P)$ is not torsion-free then $V(P)$ has a finite direct factor $K$ say $(K \neq 1)$ (see e.g., [20, p. 21]). By our remarks above $K$ is a $p$-group and hence may be taken to be cyclic of order $p^{r}$, say. Let $L$ be a complementary factor of $K$ :

$$
V(P)=L \times K \text {. }
$$

Thus $V(P) / V(P)^{r+1}$ is not a direct product of cyclic groups of order $p^{r+1}$. This means $V(P)$ is indeed torsion-free.

Now let $a \in P(a \neq 1)$. If $a \in V(P), a$ is of infinite order. If $a \notin V(P)$, observe that $a$ must be of order at least $p^{i}$ modulo $V(P)^{p^{i}}$ since in $F$ an element outside $V(F)$ is of order at least $p^{i}$ modulo $V(F)^{p^{i}}$. This last comment follows immediately by using the subgroup theorem for free groups and thinking of $F$ as $G / \gamma_{2}(V(G))$ where $G$ is an absolutely free group of rank $n$. This completes the proof of Lemma 2.

Next we prove a variation of Theorem 5.4.

LEMMA 3. Let $P$ be parafree in $\mathfrak{A} \mathfrak{B}$ where $\mathfrak{A}$ is the variety of all abelian groups and $\mathfrak{B}$ is any variety of groups. If $\left\{\mathfrak{B}_{\lambda} \mid \lambda \in \Lambda\right\}$ is the family of varieties defined by

(i) $\mathfrak{B}_{\lambda}$ is a subvariety of $\mathfrak{B}$ for every $\lambda \in \Lambda$;

(ii) there exists a prime $p$ such that $\mathfrak{B}_{\lambda}$ consists of locally finite p-groups;

(iii) every variety satisfying (i) and (ii) is in the family $\left\{\mathfrak{B}_{\lambda} \mid \lambda \in \Lambda\right\}$, then $P$ is residually parafree in $\left\{\mathfrak{U} \mathfrak{B}_{\lambda} \mid \lambda \in \Lambda\right\}$.

The proof of Lemma 3 is similar to that of Theorem 5.4. Thus suppose $a \in P$, $a \neq 1$. Since $P$ is residually nilpotent there exists a prime $p$ and a normal subgroup $N$ of $P$ such that $a \notin N$ and $P / N$ is a finite $p$-group (K. W. Gruenberg [15]). Since $P / N \in \mathfrak{A} \mathfrak{B}, V(P / N)$ is abelian. Now let $\mathfrak{U}$ be the variety generated by $P / N / V(P / N)$. Then $\mathfrak{U}$ is a variety of locally finite $p$-groups. Moreover $\mathfrak{U}$ is a subvariety of $\mathfrak{B}$. So $\mathfrak{U} \in\left\{\mathfrak{B}_{\lambda} \mid \lambda \in \Lambda\right\}$. Let $\mathfrak{W}=\mathfrak{A} \mathfrak{U}$ and put

$$
Q=P / W(P) / \gamma_{\omega}(P / W(P)) \text {. }
$$

Then of course $Q$ is residually nilpotent. Indeed we shall prove $Q$ is parafree in $\mathfrak{W}$. The argument is by now a familiar one. Thus let $F$ be free in $\mathfrak{W}$ of the same 
rank as $P$. For each $n \geqq 1, F / \gamma_{n} F$ is a quotient of $P / \gamma_{n} P$ since $\mathfrak{W} \subseteq \mathfrak{A} \mathfrak{B}$. It follows therefore from the definition of $Q$ that $F / \gamma_{n} F$ is a quotient of $Q$ and hence that $Q / \gamma_{n} Q \cong F / \gamma_{n} F$. So $Q$ is indeed parafree in $\mathfrak{W}$.

Finally we observe that as $P / N \in \mathfrak{W}, N \geqq W(P)$. But $P / N$ is nilpotent. Therefore

$$
N / W(P) \geqq \gamma_{\omega}(P / W(P)) \quad(=R / W(P), \text { say }) .
$$

Since $a \notin N, a \notin R$. So $Q \cong P / R$ is a parafree group in $\mathfrak{X} \mathfrak{U}$ in which the image of $a$ is nontrivial, where $\mathfrak{U} \in\left\{\mathfrak{B}_{\lambda} \mid \lambda \in \Lambda\right\}$. This completes the proof of Lemma 3 .

COROLLARY. An absolutely parafree group is residually parafree in $\left\{\mathfrak{A V}_{\lambda} \mid \lambda \in \Lambda\right\}$, where $\mathfrak{B}_{\lambda}$ ranges over all varieties of locally finite p-groups.

Proof. Absolutely parafree groups are residually parafree in $\left\{\mathfrak{Q}^{n} \mid n=1,2, \ldots\right\}$. For absolutely free groups are residually soluble; therefore the union of the varieties $\mathfrak{A}, \mathfrak{A}^{2}, \ldots$ is the variety of all groups. So Theorem 5.4 applies. The corollary now follows on applying Lemma 3.

We are now in a position to prove Theorem 6.1. Thus let $P$ be parafree in $\mathfrak{A B}$. By Lemma 3, $P$ is residually parafree in $\left\{\mathfrak{U}_{\mathfrak{B}_{\lambda}} \mid \lambda \in \Lambda\right\}$ where $\mathfrak{B}_{\lambda}$ ranges over varieties of locally finite $p$-groups. Hence by Lemma $2 P$ is residually torsion-free. So $P$ is torsion-free as claimed.

6.3. We recall that $\mathfrak{A}_{n}$ is the variety of all abelian groups of exponent dividing $n$. The object of this section is to prove

THEOREM 6.2. There exists continuously many nonisomorphic parafree groups of rank two in $\mathfrak{A P}_{2}$.

One consequence of the proof of Theorem 6.2 is

THEOREM 6.3. There exist a parafree group of rank two in $\mathfrak{A}_{2}$ of continuous order.

We shall need the following

LEMMA 1. Let $F$ be free in $\mathfrak{U}_{n}$ on $a$ and $b$. Then $\gamma_{2} F$ is free abelian on the elements

$$
c_{i, j}=[a, b]^{a^{i} b^{j}}, \quad 0 \leqq i, j \leqq n-1, i+j<2 n-2 .
$$

Proof. Certainly

$$
\gamma_{2} F=\operatorname{gp}\left([a, b]^{a^{b^{j}}} \mid 0 \leqq i, j \leqq n-1\right) .
$$

But

$$
1=\left[a^{n}, b^{n}\right]=[a, b]^{\left(a^{n-1}+\cdots+1\right)\left(b^{n-1}+\cdots+1\right)} .
$$

Therefore

$$
[a, b]^{a^{n-1} b^{n-1}}=[a, b]^{-\sum a^{i} b^{j}}, \quad 0 \leqq i, j<n-1, i+j<2 n-2 .
$$

We have only to check the independence of the $c_{i, j}$ 's. To do so consider the wreath product

$$
(\mathrm{gp}(\alpha) \times \mathrm{gp}(\beta)))(\mathrm{gp}(x) \times \mathrm{gp}(y))
$$

where $\alpha$ and $\beta$ are of infinite order and $x$ and $y$ are of order $n$. 
Put

$$
\alpha(i, j)=\alpha^{x^{i} y^{j}}, \beta(i, j)=\beta^{x^{i} y^{y}}
$$

Now

$$
[x \alpha, y \beta]=\alpha(0,0)^{-1} \alpha(0,1) \beta(0,0) \beta(1,0)^{-1}
$$

Thence we find

$$
[x \alpha, y \beta]^{x^{i} y^{j}}=\alpha(i, j)^{-1} \alpha(i, j+1) \beta(i, j) \beta(i+1, j)^{-1} .
$$

It follows directly on inspection of the $n^{2}-1$ commutators

$$
[x \alpha, y \beta]^{x^{i} y^{j}}, \quad 0 \leqq 1, j \leqq n-1, i+j<2 n-2
$$

that they are independent. Indeed if we list them in blocks as

$$
\begin{array}{cccc}
\alpha(0,0)^{-1} & \alpha(0,1) & \beta(0,0) & \beta(1,0)^{-1} \\
\alpha(0,1)^{-1} & \alpha(0,2) & \beta(0,1) & \beta(1,1)^{-1} \\
\vdots & \vdots & \vdots & \vdots \\
\alpha(0, n-1)^{-1} & \alpha(0,0) & \beta(0, n-1) & \beta(1, n-1)^{-1} \\
\alpha(1,0)^{-1} & \alpha(1,1) & \beta(1,0) & \beta(2,0)^{-1} \\
\alpha(1,1)^{-1} & \alpha(1,2) & \beta(1,1) & \beta(2,1)^{-1} \\
\vdots & \vdots & \vdots & \vdots \\
\alpha(1, n-1)^{-1} & \alpha(1,0) & \beta(1, n-1) & \beta(2, n-1)^{-1} \\
\vdots & \vdots & \vdots & \vdots \\
\alpha(n-1,0)^{-1} & \alpha(n-1,1) & \beta(n-1,0) & \beta(0,0)^{-1} \\
\alpha(n-1,1)^{-1} & \alpha(n-1,2) & \beta(n-1,1) & \beta(0,1)^{-1} \\
\vdots & \vdots & \vdots & \vdots \\
\alpha(n-1, n-2) & \alpha(n-1, n-1) & \beta(n-1, n-2) & \beta(0, n-2)^{-1}
\end{array}
$$

then their linear independence is immediate.

We specialize now to a free group $F$ freely generated by $a$ and $b$ in $\mathfrak{A}_{2}$. Then $\gamma_{2} F$ is free abelian on

$$
[a, b],[a, b]^{a},[a, b]^{b}
$$

and $[a, b]^{a b}=[a, b]^{-1}[a, b]^{-a}[a, b]^{-b}$.

Now let 0 be a multiplicative copy of a subgroup of the additive group of rationals which contains the integer 1 and is not divisible by 2 and let $0_{1}, 0_{2}, 0_{3}$ be indexed copies of 0 . Put

$$
N=0_{1} \times 0_{2} \times 0_{3} .
$$

Let $\mu$ be the automorphism of $N$ defined by

$$
\mu: x_{1} \mapsto x_{2}, x_{2} \mapsto x_{1}, x_{3} \mapsto x_{1}^{-1} x_{2}^{-1} x_{3}^{-1} \quad(x \in 0) .
$$

Clearly $\mu$ is of order two. Similarly let $\nu$ be the automorphism of $N$ defined by

$$
\nu: x_{1} \mapsto x_{3}, x_{2} \mapsto x_{1}^{-1} x_{2}^{-1} x_{3}^{-1}, x_{3} \mapsto x_{1} \quad(x \in 0) .
$$


Then $\nu$ too is of order two. Moreover $\mu \nu=\nu \mu$. So gp $(\mu, \nu)$ is an abelian group of order four and exponent two.

We construct now an extension $P$ of $N$ by a free abelian group $Q$ as follows:

$$
P=\operatorname{gp}\left(N, u, v ; u^{-1} w u=w \mu, v^{-1} w v=w \nu(w \in N),[u, v]=e_{1}\right) .
$$

Here $e$ stands for the element of 0 corresponding to the rational number 1 .

We compute now $\gamma_{2} P$. If $x \in 0$, then

$$
x_{1} \equiv x_{2} \equiv x_{3} \text { modulo } \gamma_{2} P \text {. }
$$

Since $x_{2} \equiv x_{1}^{-1} x_{2}^{-1} x_{3}^{-1}$ it follows that

$$
x_{1}^{4} \equiv 1 \text { modulo } \gamma_{2} P \text {. }
$$

So $N^{4}=\operatorname{gp}\left(w^{4} \mid w \in N\right) \leqq \gamma_{2} P$. But by our choice of $0,0 / 0^{4}$ is generated by $e 0^{4}$. This means that $N / N^{4}$ is generated by $e_{1}, e_{2}$ and $e_{3}$ modulo $N^{4}$. Since $e_{1} \in \gamma_{2} P$ and $e_{1} \equiv e_{2} \equiv e_{3}$ we have proved $\gamma_{2} P=N$. So $P / \gamma_{2} P$ is free abelian of rank two.

Now observe that $N / N^{2^{4}}$ is the direct product of three cyclic groups gp $\left(e_{1} N^{2^{t}}\right)$, $\mathrm{gp}\left(e_{2} N^{2^{t}}\right), \mathrm{gp}\left(e_{3} N^{2^{i}}\right)$ of order $2^{i}$. It will follow from this observation that $P$ has the same lower central sequence as the free group $F$ in $\mathfrak{U A}_{2}$ of rank two on $a$ and $b$.

It follows from Lemma 1 of 6.1 that $\gamma_{2} F / \gamma_{3} F$ is a finite 2-group since $F^{2}$ is a free abelian group on a finite number of (namely five) generators. Hence $\gamma_{2} F / \gamma_{c} F$ is a finite 2-group for every $c \geqq 2$. Therefore for some $i$,

$$
\gamma_{c} F \geqq\left(\gamma_{2} F\right)^{2^{i}} .
$$

Now inspect $F$ modulo $\left(\gamma_{2} F\right)^{2}$. It is clear then from our previous remarks and the construction of $P$ that

$$
F /\left(\gamma_{2} F\right)^{2^{i}} \cong P /\left(\gamma_{2} P\right)^{2^{4}}
$$

It follows easily that $F$ and $P$ have the same lower central sequence.

Therefore in order to prove $P$ is parafree of rank two we have only to prove that $P$ is residually nilpotent. This however is clear since

$$
\bigcap_{i=1}^{\infty} N^{2^{1}}=1
$$

and $P / N^{2^{t}}$ is nilpotent.

The proof of Theorem 6.4 is now almost complete for $\gamma_{2} P$ is the direct product of three copies of 0 , a torsion-free abelian group of rank one. By a theorem of Reinhold Baer (see e.g., [21, Vol. 1, p. 212]) this means that 0 completely determines $\gamma_{2} P$. Since the number of such 0 is the power of the continuum (see e.g., A. G. Kurosh [21, Vol. 1, p. 209]) the number of parafree groups of rank two is at least the power of the continuum. This completes the proof of Theorem 6.2.

6.4. In order to prove Theorem 6.3, let us take the group 0 in the proof of Theorem 6.1 to be a multiplicative copy of the 2 -adic integers. Then $0 / 0^{2^{i}}$ is cyclic 
of order $2^{i}$ for every $i=1,2, \ldots$ and

$$
\bigcap_{i=1}^{\infty} 0^{2^{i}}=1 \text {. }
$$

So 0 has (as a little reflection reveals) all the properties required in the proof of Theorem 6.2 which therefore goes through subject only to this modification. Since the 2-adic integers have the power of the continuum this completes the proof of Theorem 6.3.

\section{Frattini subgroups.}

7.1. The Frattini subgroup of a free group in $\mathfrak{A R}_{2}$ is trivial (J. Cossey and A. Whittemore [13]). By way of contrast we shall show that this is not always true of parafree groups in $\mathfrak{A q}_{2}$. To this end let $P$ be the group used in the proof of Theorem 6.3 where 0 is the 2-adic integers. Our aim now is to compute the Frattini subgroup of $P$. Let then $M$ be a maximal subgroup of $P$ and suppose $N=\gamma_{2} P \nsubseteq M$. Then by maximality $M N=P$. So there exist elements $u^{\prime}, v^{\prime} \in N$ such that $M \ni u u^{\prime}, v v^{\prime}$. Hence $M \cap N$ is normal in $P$. Let us put $\tilde{F}=\operatorname{gp}\left(u u^{\prime}, v v^{\prime}\right)$. Then $M=\tilde{F}(M \cap N)$. So modulo $M \cap N, \tilde{F}$ is a maximal subgroup of $P$. Therefore $N / M \cap N$ is a minimal normal subgroup of $P / M \cap N$. But observe that $\tilde{F}$ acts, by conjugation, as a finite group of automorphisms of $N / M \cap N$. Hence $N / M \cap N$ is a finitely generated abelian group and therefore finite of prime exponent $p$. But $N$ is divisible by every prime except 2. This means that $N / M \cap N$ is of exponent 2 since by assumption $M \notin N\left(=\gamma_{2} P\right)$. So $M \supseteq N^{2}$. But $P / N^{2}$ is nilpotent and every maximal subgroup of a finitely generated nilpotent group contains the derived group. So $M \supseteq N$, a contradiction. Thus we have proved $M$ does indeed contain $\gamma_{2} P$. Since $P / \gamma_{2} P$ is free abelian it follows that the Frattini subgroup of $P$ coincides with $\gamma_{2} P$.

7.2. The main result of this section is

THEOREM 7.1. Finitely generated parafree groups in $\mathfrak{A} \mathfrak{B}$ have trivial Frattini subgroup.

The main step in the proof is

Lemma 1. Let $\mathfrak{B}$ be a variety of locally finite p-groups. Then finitely generated parafree groups in $\mathfrak{A} \mathfrak{B}$ have trivial Frattini subgroup.

Proof. Let $P$ be a finitely generated parafree group in $\mathfrak{A} \mathfrak{B}$ with parabasis $x_{1}, \ldots, x_{n}$. Then

$$
Q=\operatorname{gp}\left(x_{1}, \ldots, x_{n}\right)
$$

is free in $\mathfrak{A} \mathfrak{B}$ on $x_{1}, \ldots, x_{n}$.

Now, by Theorem $6.1, P$ is torsion-free. So $V(P)$ is a free abelian group of finite rank. Moreover since $P$ has the same lower central sequence as $Q, V(Q)$ is a free abelian group of the same rank as $V(P)$. So $V(P) / V(Q)$ is finite of order $m$. (Actually $m$ is prime to $p$.) So if $q$ is any prime which is different from $p$ and does not divide $m$, then, modulo $V(P)^{q}, V(Q)$ coincides with $V(P)$. Moreover

$$
V(P)^{q} \cap V(Q)=V(Q)^{q} .
$$


So

$$
P / V(P)^{q}=Q V(P)^{q} / V(P)^{q} \cong Q / V(Q)^{q} .
$$

Therefore $P / V(P)^{q}$ is free in $\mathfrak{A}_{q} \mathfrak{B}$ where $\mathfrak{A}_{q}$ is, of course, the variety of all abelian groups of exponent $q$. But the Frattini subgroup of such a group is trivial (see e.g., J. Cossey and A. Whittemore [13]). Therefore

$$
\phi(P) \subseteq V(P)^{q} .
$$

Hence

$$
\phi(P) \subseteq \bigcap V(P)^{q}
$$

where this intersection ranges over all primes $q$ which do not divide $m$ and are different from $p$. Consequently $\phi(P)=1$. This completes the proof of Lemma 1 .

Theorem 7.1 follows easily from Lemma 1 . Indeed if $P$ is finitely generated and parafree in $\mathfrak{A} \mathfrak{B}$ then $P$ is residually parafree in $\left\{\mathfrak{A}_{\mathfrak{B}_{\lambda}} \mid \lambda \in \Lambda\right\}$ where $\mathfrak{B}_{\lambda}$ ranges over a family of varieties of locally finite $p$-groups (Lemma 3 of 6.1 ). So $\phi P$ is trivial, by Lemma 1 .

Since, by the corollary to Lemma 3 of 6.1 , absolutely parafree groups are residually parafree in $\left\{\mathfrak{U}_{\lambda} \mid \lambda \in \Lambda\right\}$ where $\mathfrak{B}_{\lambda}$ ranges e.g., over a family of locally finite $p$-groups, it follows immediately from Theorem 7.1 that

COROLLARY 1. Finitely generated absolutely parafree groups have trivial Frattini subgroups.

Similarly one has

COROLlaRY 2. A finitely generated parafree group in the variety of all solvable groups of derived length at most $n$ has trivial Frattini subgroup.

\section{REFERENCES}

1. Maurice Auslander and R. C. Lyndon, Commutator subgroups of free groups, Amer. J. Math. 77 (1955), 929-931.

2. S. Bachmuth, Automorphisms of free metabelian groups, Trans. Amer. Math. Soc. 118 (1965), 93-104.

3. G. Baumslag, Groups with the same lower central sequence as a relatively free group. I. The groups, Trans. Amer. Math. Soc. 129 (1967), 308-321.

4. - Some groups which are just about free, Bull. Amer. Math. Soc. 73 (1967), 621-622.

5. - More groups which are just about free, Bull. Amer. Math. Soc. 74 (1968), 752-754.

6. - Some subgroup theorems for free $\mathfrak{v}$-groups, Trans. Amer. Math. Soc. 108 (1963), 516-525.

7. - Wreath products and extensions, Math. Z. 81 (1963), 286-299.

8. - , A representation of the wreath product of two torsion-free abelian groups in a graded ring, Proc. Amer. Math. Soc. 17 (1966), 1159-1165.

9. - On the residual nilpotence of certain one-relator groups, Comm. Pure Appl. Math. 21 (1968), 491-506.

10. A. H. Clifford and G. B. Preston, The algebraic theory of semigroups, Vols. 1, 2, Math. Surveys, No. 7, Amer. Math. Soc., Providence, R. I., 1961, 1967. 
11. D. E. Cohen, On the laws of a metabelian variety, J. Algebra 5 (1967), 267-273.

12. P. M. Cohn, Factorization in non-commutative power series rings, Proc. Cambridge Philos. Soc. 58 (1962), 449-464.

13. P. J. Cossey and A. Whittemore, On Frattini subgroups, (to appear).

14. S. Eilenberg and N. Steenrod, Foundations of algebraic topology, Princeton University Press, Princeton, N. J., 1952.

15. K. W. Gruenberg, Residual properties of infinite soluble groups, Proc. London Math. Soc. (3) 7 (1957), 29-62.

16. G. Higman, Finite groups having isomorphic images in every finite group of which they are homomorphic images, Quart. J. Math. 6 (1955), 250-254.

17. G. Higman, B. H. Neumann and Hanna Neumann, Embedding theorems for groups, J. London Math. Soc. 26 (1951), 59-61.

18. G. Higman and B. H. Neumann, On two questions of Ito, J. London Math. Soc. 29 (1954), 84-88.

19. N. Jacobson, Lie algebras, Interscience, New York, 1961.

20. I. Kaplansky, Infinite abelian groups, Univ. of Michigan, Ann Arbor, Mich., 1954.

21. A. G. Kurosh, The theory of groups, Vols. I, II, transl. by K. A. Hirsch, Chelsea, New York, 1955.

22. M. Lazard, Groupes analytiques p-adiques, Inst. Hautes Études Sci. Publ. Math. No. 26 (1965).

23. W. Magnus, Beziehungen zwischen Gruppen und Idealen in einem speziellen Ring, Math. Ann. 111 (1935), 259-280.

24. - On a theorem of M. Hall, Ann. of Math. 40 (1939), 764-768.

25. W. Magnus, A. Karrass and D. Solitar, Combinatorial group theory, Interscience, New York, 1966.

26. A. I. Mal'cev, Generalized nilpotent groups and their adjoint groups, Mat. Sb. 25 (1949), 347-366. (Russian)

27. S. Meskin, Ph.D. Thesis, City University of New York, 1968.

28. S. Moran, Associative operations in groups. I, Proc. London Math. Soc. (3) 6 (1956), 581-596.

29. H. Neumann, Varieties of groups, Ergebnisse der Mathematik und ihrer Grenzgebiete Bd. 37, Springer-Verlag, New York, 1967.

30. J. Nielsen, Die Isomorphismengruppe der freien Gruppen, Math. Ann. 91 (1924), 169-209.

31. J. Ridley, Ph.D. Thesis, London University, 1967.

32. H. Seifert and W. Threlfall, Lehrbuch der Topologie, Chelsea, New York, 1947.

33. J. H. C. Whitehead, On equivalent sets of elements in a free group, Ann. of Math. 37 (1963), 782-800.

34. E. Witt, Treue Darstellung Liescher Ringe, J. Reine Angew. Math. 117 (1937), 152-160.

Graduate Center, City University of New York, New York, New YorK 